\title{
Present Status and Future Perspectives of the NEXT Experiment
}

\author{
J. J. Gómez Cadenas, ${ }^{1}$ V. Álvarez, ${ }^{1}$ F. I. G. Borges, ${ }^{2}$ S. Cárcel, ${ }^{1}$ J. Castel, ${ }^{3}$ S. Cebrián, ${ }^{3}$
} A. Cervera, ${ }^{1}$ C. A. N. Conde, ${ }^{2}$ T. Dafni, ${ }^{3}$ T. H. V. T. Dias, ${ }^{2}$ J. Díaz, ${ }^{1}$ M. Egorov, ${ }^{4}$ R. Esteve, ${ }^{5}$ P. Evtoukhovitch ${ }^{6}$ L. M. P. Fernandes, ${ }^{2}$ P. Ferrario, ${ }^{1}$ A. L. Ferreira, ${ }^{7}$ E. D. C. Freitas, ${ }^{2}$ V. M. Gehman, ${ }^{4}$ A. Gil, ${ }^{1}$ A. Goldschmidt, ${ }^{4}$ H. Gómez, ${ }^{3}$ D. González-Díaz, ${ }^{3}$ R. M. Gutiérrez, ${ }^{8}$ J. Hauptman, ${ }^{3}$ J. A. Hernando Morata, ${ }^{10}$ D. C. Herrera, ${ }^{3}$ F. J. Iguaz, ${ }^{3}$ I. G. Irastorza, ${ }^{3}$ M. A. Jinete,${ }^{8}$ L. Labarga, ${ }_{11}^{11}$ A. Laing, ${ }^{1}$ I. Liubarsky, ${ }^{1}$ J. A. M. Lopes, ${ }^{2}$ D. Lorca, ${ }^{1}$ M. Losada, ${ }^{8}$ G. Luzón, ${ }^{3}$ A. Marí, ${ }^{5}$ J. Martín-Albo, ${ }^{1}$ A. Martínez, ${ }^{1}$ T. Miller, ${ }^{4}$ A. Moiseenko, ${ }^{6}$ F. Monrabal, ${ }^{1}$ M. Monserrate, ${ }^{1}$ C. M. B. Monteiro, ${ }^{2}$ F. J. Mora, ${ }^{5}$ L. M. Moutinho, ${ }^{7}$ J. Muñoz Vidal, ${ }^{1}$ H. Natal da Luz, ${ }^{2}$ G. Navarro, ${ }^{8}$ M. Nebot-Guinot, ${ }^{1}$ D. Nygren, ${ }^{4}$ C. A. B. Oliveira, ${ }^{4}$ R. Palma, ${ }^{12}$ Javier Pérez, ${ }^{13}$ J. L. Pérez-Aparicio, ${ }^{12}$ J. Renner, ${ }^{4}$ L. Ripoll, ${ }^{14}$ A. Rodríguez, ${ }^{3}$ J. Rodríguez, ${ }^{1}$ F. P. Santos, ${ }^{2}$ J. M. F. dos Santos, ${ }^{2}$ L. Segui, ${ }^{3}$ L. Serra, ${ }^{1}$ D. Shuman, ${ }^{4}$ A. Simón, ${ }^{1}$ C. Sofka, ${ }^{15}$ M. Sorel, ${ }^{1}$ J. F. Toledo, ${ }^{5}$ A. Tomás, ${ }^{3}$ J. Torrent, ${ }^{14}$ Z. Tsamalaidze, ${ }^{6}$ J. F. C. A. Veloso, ${ }^{7}$ J. A. Villar, ${ }^{3}$ R. Webb, ${ }^{15}$ J. White, ${ }^{15}$ and N. Yahlali ${ }^{1}$

\footnotetext{
${ }^{1}$ Instituto de Física Corpuscular (IFIC), CSIC-Universidad de Valencia, Calle Catedrático José Beltrán 2, Paterna, 46980 Valencia, Spain

${ }^{2}$ Departamento de Fisica, Universidad de Coimbra, Rua Larga, 3004-516 Coimbra, Portugal

${ }^{3}$ Laboratorio de Física Nuclear y Astropartículas, Universidad de Zaragoza, Calle Pedro Cerbura 12, 50009 Zaragoza, Spain

${ }^{4}$ Lauwrence Berkeley National Laboratory (LBNL), 1 Cyclotron Road, Berkeley, CA 94720, USA

${ }^{5}$ Instituto de Instrumentación para Imagen Molecular (I3M), UPV, Camino de Vera $s / n$, Edificio $8 B$, 46022 Valencia, Spain

${ }^{6}$ Joint Institute for Nuclear Research (JINR), Joliot-Curie 6, Dubna 141980, Russia

${ }^{7}$ Institute of Nanostructures, Nanomodelling and Nanofabrication (I3N), Universidad de Aviero, Campus de Santiago, 3810-193 Aveiro, Portugal

${ }^{8}$ Centro de Investigaciones, Universidad Antonio Nariño, Carretera 3 Este No. 47A-15, Bogota, Colombia

${ }^{9}$ Department of Physics and Astronomy, Iowa State University, 12 Physics Hall, Ames, IA 50011-3160, USA

${ }^{10}$ Instituto Gallego de Física de Altas Energías (IGFAE), Universidad de Santiago de Compostela, Campus Sur, Rua Xosé María Suarez Nuñez s/n, Campus Vida, 15782 Santiago de Compostela, Spain

${ }^{11}$ Departamento de Física Teórica, Universidad Autónoma de Madrid, Campus de Cantoblanco, 28049 Madrid, Spain

${ }^{12}$ Departamento de Mecánica de Medios Continuos y Teoría de Estructuras, Universidad Politécnica de Valencia, Camino de Vera s/n, 46071 Valencia, Spain

${ }^{13}$ Instituto de Física Teórica (CSIC), UAM, Campus Cantoblanco, 28049 Madrid, Spain

${ }^{14}$ Escola Politécnica Superior, Universitat de Girona, Avenida Montilivi s/n, 17071 Girona, Spain

${ }^{15}$ Departament of Physics and Astronomy, Texas A \& M University, College Station, TX 77843-4242, USA
}

Correspondence should be addressed to P. Ferrario; paola.ferrario@ific.uv.es

Received 12 July 2013; Accepted 9 October 2013; Published 18 March 2014

Academic Editor: Vincenzo Flaminio

Copyright (C) 2014 J. J. Gómez Cadenas et al. This is an open access article distributed under the Creative Commons Attribution License, which permits unrestricted use, distribution, and reproduction in any medium, provided the original work is properly cited. The publication of this article was funded by SCOAP ${ }^{3}$. 
NEXT is an experiment dedicated to neutrinoless double beta decay searches in xenon. The detector is a TPC, holding $100 \mathrm{~kg}$ of high-pressure xenon enriched in the ${ }^{136} \mathrm{Xe}$ isotope. It is under construction in the Laboratorio Subterráneo de Canfranc in Spain, and it will begin operations in 2015. The NEXT detector concept provides an energy resolutionbetter than 1\% FWHM and a topological signal that can be used to reduce the background. Furthermore, the NEXT technology can be extrapolated to a 1 ton-scale experiment.

In memoriam of our friend and mentor James White

\section{Introduction}

This paper presents the current status and future prospects of the Neutrino Experiment with a Xenon TPC (NEXT) (http://next.ific.uv.es/next). The primary goal of the project is the construction, commissioning, and operation of the NEXT-100 detector, a high-pressure xenon (HPXe) time projection chamber (TPC). NEXT-100 will search for neutrinoless double beta decay $(\beta \beta 0 \nu)$ events using $100 \mathrm{~kg}$ of xenon enriched at $90 \%$ in the isotope ${ }^{136} \mathrm{Xe}$. The experiment will operate at the Laboratorio Subterráneo de Canfranc (LSC), starting in 2015. The NEXT collaboration includes institutions from Spain, Portugal, USA, Russia, and Colombia.

The discovery potential of a HPXe TPC combines four desirable features that make it an almost-ideal experiment for $\beta \beta 0 v$ searches, namely,

(1) excellent energy resolution (0.5-0.7\% FWHM in the region of interest),

(2) a topological signature (the observation of the tracks of the two electrons),

(3) a fully active, very radiopure apparatus of large mass,

(4) the capability of extending the technology to a tonscale experiment,

Currently, two xenon-based experiments, with a mass in the range of hundred kilograms, are dominating the field of $\beta \beta 0 v$ searches. These are EXO-200 (a liquid xenon TPC) [1] and KamLAND-Zen (a large, liquid scintillator calorimeter, where xenon is dissolved in the scintillator) [2]. NEXT features a better resolution and the extra handle of the identification of the two electrons, which could result in a discovery, in spite of a late start. If evidence is found by EXO200 or KamLAND-Zen of the existence of a signal, NEXT would be ideally suited to confirm it in an unambiguous way, in particular given the discriminating power of the topological signature.

The negative results of EXO-200 and KamLAND-Zen indicate that the effective neutrino mass (the quantity measured in $\beta \beta$ decays, as further discussed later in the text) must be smaller than $120-250 \mathrm{meV}$, where the mass range is due to uncertainties in the nuclear matrix elements. On the other hand, recent measurements of the cosmic microwave background (CMB) by the Planck experiment $[3,4]$ yield an upper limit for the sum of the three light neutrino masses of $230 \mathrm{meV}$. The latter result excludes most of the so-called degenerate spectrum, in which the three neutrino masses are relatively large and similar to each other. The current sensitivity of the $\beta \beta 0 \nu$ experiments is not enough to explore significantly the so-called inverse hierarchy, which require sensitivities to effective neutrino masses in the range of $20 \mathrm{meV}$ (if nature has chosen the so-called normal hierarchy as her preferred pattern for neutrino masses the search for $\beta \beta 0 \nu$ processes becomes extremely difficult if not hopeless). It follows that the next generation of $\beta \beta 0 \nu$ experiments must improve their sensitivity by typically one order of magnitude in the effective neutrinos mass, or two orders of magnitude in the period of the $\beta \beta 0 \nu$ decay. This, in turn, requires increasing by a factor 100 the exposure from the "typical" values of the current generation of experiments (thus going from $\sim 100 \mathrm{~kg}$ per year to $\sim$ one ton per 10 years), while at the same time decreasing by a factor 100 the residual backgrounds (e.g., going from few events per $100 \mathrm{~kg}$ to $\sim 0.1$ events per ton).

This tremendous challenge requires a detector capable to deploy a large source mass of pure isotope at a reasonable cost. Currently, only xenon has demonstrated this capability. There is already more than one ton of enriched xenon in the world, owned by KamLAND-Zen (800 kg), EXO-200 $(200 \mathrm{~kg})$, and NEXT $(100 \mathrm{~kg})$. Furthermore, xenon detectors are fully active (the detection medium is the same as the isotope source) and scalable, being either TPCs (EXO-200, NEXT) or scintillating calorimeters with the xenon dissolved in the scintillator (KamLAND-Zen).

The physics case of a HPXe TPC is outstanding, given the combination of excellent energy resolution and the high background rejection power that the observation of the two electrons provides. In that respect, NEXT-100 will serve also as a springboard for the next generation of ton-scale, HPXe experiments.

This paper is organised as follows. Section 2 describes the physics of Majorana neutrinos and $\beta \beta 0 \nu$ searches, reviews xenon experiments, and discusses their discovery potential. The NEXT detector is described with some details in Section 3, while Section 4 gives details of the NEXT background model. Section 5 describes our two electroluminescence prototypes, NEXT-DEMO and NEXT-DBDM. Finally, conclusions are presented in Section 6.

\section{The Physics of NEXT}

2.1. Majorana Neutrinos and $\beta \beta 0 \nu$ Experiments. Neutrinos, unlike the other Standard Model fermions, could be truly neutral particles, that is, indistinguishable from their antiparticles. The existence of such Majorana neutrinos would imply the existence of a new energy scale of physics that characterizes new dynamics beyond the Standard Model and provides the simplest explanation of why neutrino masses 


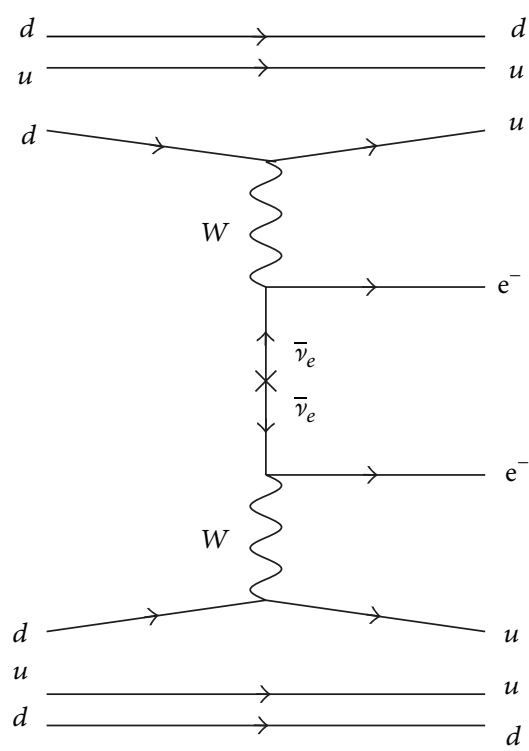

FIGURE 1: Feynman diagram for $\beta \beta 0 \nu$ through the exchange of a light Majorana neutrino.

are so much lighter than the charged fermions. (The term Majorana neutrino honors the Italian physicist Majorana, who, in 1937, published a fundamental paper [5] in which he was able "to build a substantially novel theory for the particles deprived of electric charge." Even if in those times the only known "charge" was the electric charge, the Majorana implicitly assumed particles deprived of all the possible charges. In modern language, neutrinos should not have any lepton number. In other words, Majorana theory describes completely neutral spin $1 / 2$ particles, which are identical to their antiparticles.) Understanding the new physics that underlies neutrino masses is one of the most important open questions in particle physics. It could have profound implications in our understanding of the mechanism of symmetry breaking, the origin of mass, and the flavor problem [6].

Furthermore, the existence of Majorana neutrinos would imply that lepton number is not a conserved quantum number. This, in turn, could be the origin of the matterantimatter asymmetry observed in the universe. The new physics related to neutrino masses could provide a new mechanism to generate that asymmetry, called leptogenesis $[7,8]$. Although the predictions are model dependent, two essential ingredients must be confirmed experimentally: (1) the violation of lepton number and (2) CP violation in the lepton sector.

The only practical way to establish experimentally that neutrinos are their own antiparticle is the detection of neutrinoless double beta decay $(\beta \beta 0 \nu)$. This is a postulated very slow radioactive process in which a nucleus with $Z$ protons decays into a nucleus with $Z+2$ protons and the same mass number $A$, emitting two electrons that carry essentially all the energy released $\left(Q_{\beta \beta}\right)$. The process can occur if and only if neutrinos are massive, Majorana particles.

Several underlying mechanisms-involving physics beyond the Standard Model-have been proposed for $\beta \beta 0 v$, the simplest one being the virtual exchange of light Majorana neutrinos, shown in Figure 1. Assuming this to be the dominant process at low energies, the half-life of $\beta \beta 0 \nu$ can be written as

$$
\left(T_{1 / 2}^{0 v}\right)^{-1}=G^{0 v}\left|M^{0 v}\right|^{2} m_{\beta \beta}^{2}
$$

In this equation, $G^{0 v}$ is an exactly calculable phase-space integral for the emission of two electrons; $M^{0 v}$ is the nuclear matrix element (NME) of the transition, which has to be evaluated theoretically; and $m_{\beta \beta}$ is the effective Majorana mass of the electron neutrino:

$$
m_{\beta \beta}=\left|\sum_{i} U_{e i}^{2} m_{i}\right|,
$$

where $m_{i}$ are the neutrino mass eigenstates and $U_{e i}$ are elements of the neutrino mixing matrix. Therefore, a measurement of the decay rate of $\beta \beta 0 \nu$ would provide direct information on neutrino masses.

The relationship between $m_{\beta \beta}$ and the actual neutrino masses $m_{i}$ is affected by the uncertainties in the measured oscillation parameters, the unknown neutrino mass ordering (normal or inverted), and the unknown phases in the neutrino mixing matrix. The current knowledge on neutrino masses and mixings provided by neutrino oscillation experiments is summarised in Figure 2(a). The diagram shows the two possible mass orderings that are compatible with neutrino oscillation data, with increasing neutrino masses from bottom to top. The relationship between $m_{\beta \beta}$ and the lightest neutrino mass $m_{\text {light }}$ (which is equal to $m_{1}$ or $m_{3}$ in the normal and inverted mass orderings, resp.) is illustrated in Figure 2(b).

The upper bound on the effective Majorana mass corresponds to the experimental constraint set by the HeidelbergMoscow (HM) experiment, which was until very recently the most sensitive limit to the half-life of $\beta \beta 0 v: T_{1 / 2}^{0 \nu}\left({ }^{76} \mathrm{Ge}\right) \geq 1.9 \times$ $10^{25}$ years at $90 \%$ CL [9]. A subgroup of the HM experiment interpreted the data as evidence of a positive signal, with a best value for the half-life of $2.23 \times 10^{25}$ years, corresponding to an effective Majorana mass of about $300 \mathrm{meV}$ [10]. This claim was very controversial and the experimental effort of the last decade has been focused on confirming or refuting it. The recent results from the KamLAND-Zen and EXO experiments have almost excluded the claim, and new data from other experiments such as GERDA [11], MAJORANA [12], and CUORE [13] will definitively settle the question shortly.

2.2. The Current Generation of $\beta \beta 0 v$ Experiments. The detectors used to search for $\beta \beta 0 \nu$ are designed, in general, to measure the energy of the radiation emitted by a $\beta \beta 0 v$ source. In a neutrinoless double beta decay, the sum of the kinetic energies of the two released electrons is always the same and equal to the mass difference between the parent and the daughter nuclei: $Q_{\beta \beta} \equiv M(Z, A)-M(Z+2, A)$. However, due to the finite energy resolution of any detector, $\beta \beta 0 v$ events would be reconstructed within a given energy range centred around $Q_{\beta \beta}$ and typically following a Gaussian distribution. Other processes occurring in the detector can 


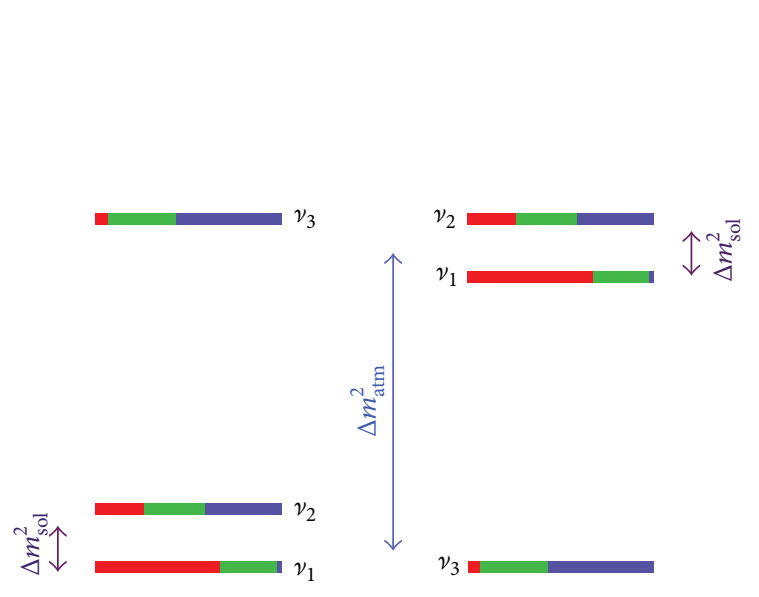

(a)

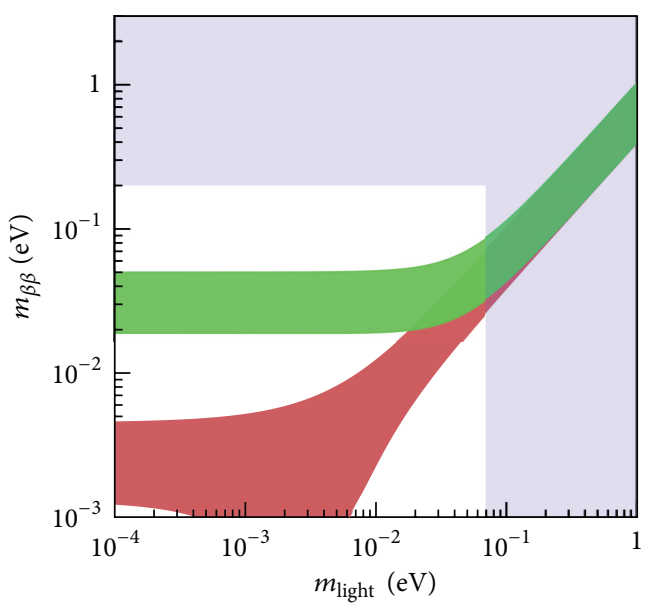

(b)

FIGURE 2: The left image shows the normal (a) and inverted (b) mass orderings. The electron, muon, and tau flavor content of each neutrino mass eigenstate are shown via the red, green, and blue fractions, respectively. $\Delta m_{\text {sol }}^{2} \equiv m_{2}^{2}-m_{1}^{2}$, while $\Delta m_{\text {atm }}^{2} \equiv m_{3}^{2}-m_{1}^{2}$ in the normal ordering and $\Delta m_{\mathrm{atm}}^{2} \equiv m_{3}^{2}-m_{2}^{2}$ in the inverted ordering. The right plot shows the effective neutrino Majorana mass, $m_{\beta \beta}$, as a function of the lightest neutrino mass, $m_{\text {light }}$. The green band corresponds to the inverse hierarchy of neutrino masses, whereas the red corresponds to the normal ordering. The upper bound on the lightest neutrino mass comes from cosmological bounds; the bound on the effective Majorana mass comes from $\beta \beta 0 \nu$ constraints.

fall in that region of energies, thus becoming a background and compromising the sensitivity of the experiment [14].

All double beta decay experiments have to deal with an intrinsic background, the standard two-neutrino double beta decay $(\beta \beta 2 \nu)$, which can only be suppressed by means of good energy resolution. Backgrounds of cosmogenic origin force the underground operation of the detectors. Natural radioactivity emanating from the detector materials and surroundings can easily overwhelm the signal peak, and hence careful selection of radiopure materials is essential. Additional experimental signatures, such as event topological information, which allow the distinction of signal and background, are a bonus to provide a robust result.

Besides energy resolution and control of backgrounds, several other factors such as detection efficiency and scalability to large masses must be taken into consideration in the design of a double beta decay experiment. The simultaneous optimisation of all these parameters is most of the time conflicting, if not impossible, and consequently many different experimental techniques have been proposed. In order to compare them, a figure of merit, the experimental sensitivity to $m_{\beta \beta}$, is normally used [14]:

$$
m_{\beta \beta} \propto \sqrt{\frac{1}{\varepsilon}}\left(\frac{b \delta E}{M t}\right)^{1 / 4}
$$

where $\varepsilon$ is the signal detection efficiency, $M$ is the $\beta \beta$ isotope mass used in the experiment, $t$ is the data-taking time, $\delta E$ is the energy resolution, and $b$ is the background rate in the region of interest around $Q_{\beta \beta}$ (expressed in counts per $\mathrm{kg}$ of $\beta \beta$ isotope, year, and $\mathrm{keV}$ ).

Among the ongoing and planned experiments, many different experimental techniques are utilised, each with its pros and cons. The time-honored approach of emphasising energy resolution and detection efficiency is currently spearheaded by germanium calorimeters like GERDA and Majorana, as well as tellurium bolometers such as CUORE. Other experiments that will operate in the next few years are the tracker-calorimeter SuperNEMO demonstrator [15] and the liquid scintillator calorimeter $\mathrm{SNO}+[16]$, in which the isotope is dissolved in the scintillator. For a detailed discussion of the status of the field we refer the reader to several recent reviews [15, 17-20].

A different and powerful approach proposes the use of xenon-based experiments. Two of them, EXO-200 [21] and KamLAND-Zen [22], are already operating, as mentioned in Section 1, while NEXT [23] is in the initial stages of construction and is foreseen to start taking data in 2015.

2.3. Xenon Experiments. Xenon is an almost-optimal element for $\beta \beta 0 v$ searches, featuring many desirable properties, both as a source and as a detector. It has two naturally occurring isotopes that can decay via the $\beta \beta$ process, ${ }^{134} \mathrm{Xe}\left(\mathrm{Q}_{\beta \beta}=\right.$ $825 \mathrm{keV})$ and ${ }^{136} \mathrm{Xe}\left(Q_{\beta \beta}=2458 \mathrm{keV}\right)$. The latter, having a higher $Q$ value, is preferred since the decay rate is proportional to $Q_{\beta \beta}^{5}$ and the radioactive backgrounds are less abundant at higher energies. Moreover, the $\beta \beta 2 v$ mode of ${ }^{136} \mathrm{Xe}$ is slow $\left(\sim 2.3 \times 10^{21}\right.$ years $\left.[22,24]\right)$ and hence the experimental requirement for good energy resolution to suppress this particular background is less stringent than for other $\beta \beta$ sources. The process of isotopic enrichment in the isotope ${ }^{136} \mathrm{Xe}$ is relatively simple and cheap compared to that of other $\beta \beta$ isotopes. Xenon has no long-lived radioactive isotopes and, being a noble gas, can be easily purified.

As a detector, xenon is a noble gas, and therefore one can build a time projection chamber (TPC) with pure xenon as 
detection medium. Both a liquid xenon (LXe) TPC and a (high-pressure) gas (HPXe) TPC are suitable technologies. Nevertheless, energy resolution is much better in gas than in liquid, since, in its gaseous phase, xenon is characterized by a small Fano factor, meaning that the fluctuations in the ionization production are smaller than the ones due to pure Poisson statistics. The largest HPXe chamber ever operated in the world before NEXT was the Gotthard TPC [25]. It had a total mass of $5 \mathrm{~kg}$ of xenon and a pressure of $\sim 5 \mathrm{bar}$. The technology of the time (the Gotthard experiment was being run in the mid-90s) allowed achieving a modest energy resolution (around 7\%). The two current experiments using xenon are the EXO-200 collaboration, which has chosen the LXe approach, and the KamLAND-Zen experiment, which dissolves the isotope in liquid scintillator.

2.3.1. EXO. The EXO-200 detector [21] is a symmetric LXe TPC deploying $110 \mathrm{~kg}$ of xenon (enriched to $80.6 \%$ in ${ }^{136} \mathrm{Xe}$ ).

In EXO-200, ionization charges created in the xenon by charged particles drift under the influence of an electric field towards the two ends of the chamber. There, the charge is collected by a pair of crossed wire planes which measure its amplitude and transverse coordinates. Each end of the chamber includes also an array of avalanche photodiodes (APDs) to detect the $178 \mathrm{~nm}$ scintillation light. The sides of the chamber are covered with Teflon sheets that act as VUV reflectors, improving the light collection. The simultaneous measurement of both the ionisation charge and scintillation light of the event may in principle allow reaching a detector energy resolution as low as 3.3\% FWHM at the ${ }^{136} \mathrm{Xe} Q$ value, for a sufficiently intense drift electric field [26].

The EXO-200 detector achieves currently an energy resolution of $4 \% \mathrm{FWHM}$ at $Q_{\beta \beta}$ and a background rate of $1.5 \times 10^{-3}$ counts/(keV. $\left.\mathrm{kg} \cdot \mathrm{y}\right)$ in the region of interest (ROI). The region of interest is the energy interval around the $Q_{\beta \beta}$ corresponding to the resolution achieved by an experiment, inside which it is impossible to distinguish the background from the signal. The experiment has also searched for $\beta \beta 0 v$ events. The total exposure used for the published result is $32.5 \mathrm{~kg}$.year. They have published a limit on the half-life of $\beta \beta 0 \nu$ of $T_{1 / 2}^{0 \nu}\left({ }^{136} \mathrm{Xe}\right)>1.6 \times 10^{25}$ years $[1]$.

2.3.2. KamLAND-Zen. The KamLAND-Zen experiment is a modification of the well-known KamLAND neutrino detector [22]. A transparent balloon, with a $\sim 3 \mathrm{~m}$ diameter, containing 13 tons of liquid scintillator loaded with $320 \mathrm{~kg}$ of xenon (enriched to $91 \%$ in ${ }^{136} \mathrm{Xe}$ ) is suspended at the centre of KamLAND. The scintillation light generated by events occurring in the detector is recorded by an array of photomultipliers surrounding it. The position of the event vertex is reconstructed with a spatial resolution of about $15 \mathrm{~cm} / \sqrt{E(\mathrm{MeV})}$. The energy resolution is $(6.6 \pm$ $0.3) \% / \sqrt{E(\mathrm{MeV})}$, that is, 9.9\% FWHM at the $Q$ value of

${ }^{136} \mathrm{Xe}$. The signal detection efficiency is $\sim 0.42$ due to the tight fiducial cut introduced to reject backgrounds originating in the balloon. The achieved background rate in the energy window between $2.2 \mathrm{MeV}$ and $3.0 \mathrm{MeV}$ is $10^{-3}$ counts/(keV·kg.y).

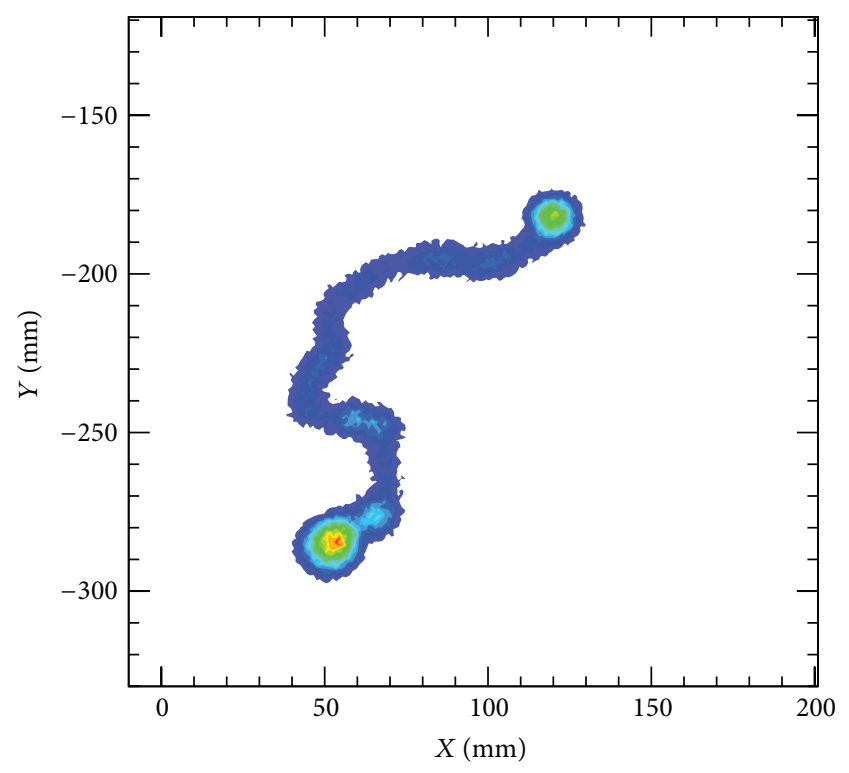

FIgUre 3: Monte Carlo simulation of $\mathrm{a}^{136} \mathrm{Xe} \beta \beta 0 \nu$ event in xenon gas at 10 bar: the ionization track, about $30 \mathrm{~cm}$ long, is tortuous because of multiple scattering and has larger depositions or blobs in both ends.

KamLAND-Zen has searched for $\beta \beta 0 \nu$ events with an exposure of $89.5 \mathrm{~kg} \cdot y e a r$. They have published a limit on the half-life of $\beta \beta 0 \nu$ of $T_{1 / 2}^{0 \nu}\left({ }^{136} \mathrm{Xe}\right)>1.9 \times 10^{25}$ years [2].

The combination of the KamLAND-Zen and EXO results yields a limit $T_{1 / 2}^{0 v}\left({ }^{136} \mathrm{Xe}\right)>3.4 \times 10^{25}$ years $(120-250 \mathrm{meV}$, depending on the NME) [2], which essentially excludes the long-standing claim of Klapdor-Kleingrothaus and collaborators [27].

2.3.3. NEXT: A Preview. The NEXT experiment will search for $\beta \beta 0 v$ in ${ }^{136} \mathrm{Xe}$ using a high-pressure xenon gas (HPXe) time projection chamber (TPC) containing 100 kilograms of enriched gas, and called NEXT-100. Such a detector offers major advantages for the search of neutrinoless double beta decay as follows.

(i) Excellent energy resolution, with an intrinsic limit of about $0.3 \%$ FWHM at $Q_{\beta \beta}$ and $0.5-0.7 \%$ demonstrated by the NEXT prototypes, as explained in detail in Sections 5.1 and 5.2. For reference, the best energy resolution in the field is achieved by germanium experiments, such as GERDA and MAJORANA, or bolometers such as CUORE, with typical resolutions in the range of $0.2 \% \mathrm{FWHM}$ at $Q_{\beta \beta}$. NEXT-100 targets a resolution which is a factor two worse than these but a factor 8 (20) better than that of EXO (KamLANDZen), the other xenon experiments $[1,2]$.

(ii) Tracking capabilities that provide a powerful topological signature to discriminate between signal (two electron tracks with a common vertex) and background (mostly, single electrons). Neutrinoless double beta decay events leave a distinctive topological signature in gaseous xenon: an ionization track, about $30 \mathrm{~cm}$ 


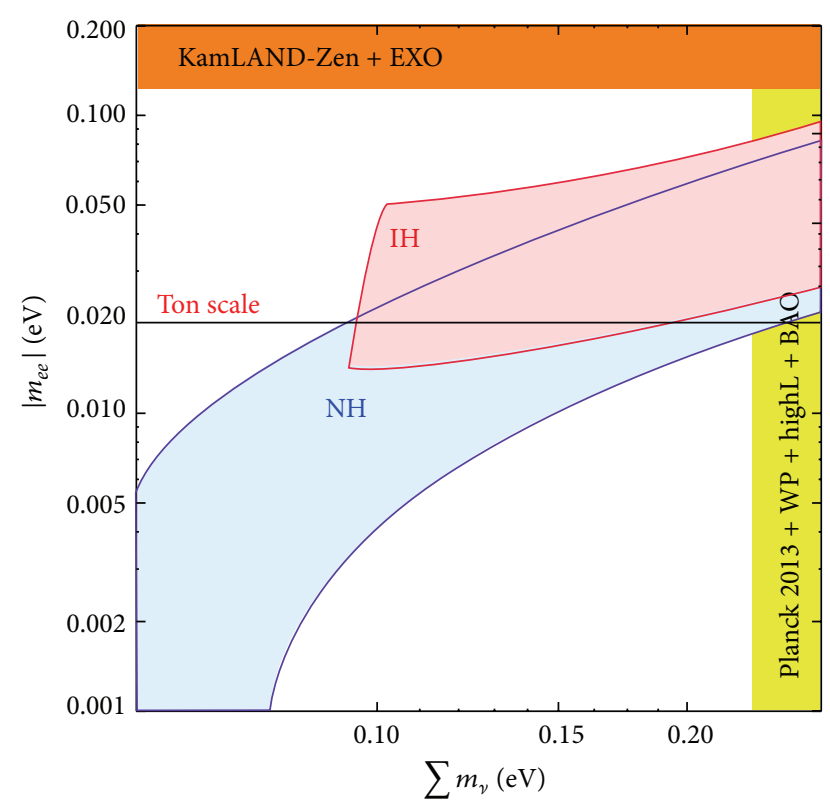

FIgURE 4: The cosmological constraint on the sum of the neutrino mass derived from Planck combined with other data, together with the best limits from $\beta \beta 0 v$ experiments (KamLAND-Zen + EXO) and the expected limit that can be reached by the high-performing experiments in the ton scale, in particular NEXT. $\left|m_{e e}\right|$ is the effective Majorana mass defined in (2), and $\mathrm{IH}$ and $\mathrm{NH}$ stand for inverted and normal hierarchy, respectively. Adapted from [4].

long at $10 \mathrm{bar}$, tortuous due to multiple scattering, and with larger energy depositions at both ends (see Figure 3). The Gotthard experiment proved the effectiveness of such a signature to discriminate signal from background. The topological signature results in an expected background rate of the order of $5 \times 10^{-4}$ counts/(keV·kg.y), improving EXO and KamLAND-Zen by a factor two $[1,2]$ and the germanium calorimeters and tellurium bolometers by a factor five to ten.

(iii) A fully active and homogeneous detector. Since 3dimensional reconstruction is possible, events can be located in a fiducial region away from surfaces, where most of the background arises. This is a common feature with the two other xenon experiments.

(iv) Scalability of the technique to larger masses, thanks to the fact that: (a) xenon is noble gas, suitable for detection and with no intrinsic radioactivity; (b) enriched xenon (in Xe-136) can be procured at a moderately limited cost, for instance a factor 10 cheaper than the baseline ${ }^{76} \mathrm{Ge}$ choice. This is also a common feature with the other two xenon experiments.

2.4. Discovery Potential of Xenon Experiments. Recently, an upper limit at $95 \%$ confidence level for the sum of the three light neutrino masses has been reported by Planck

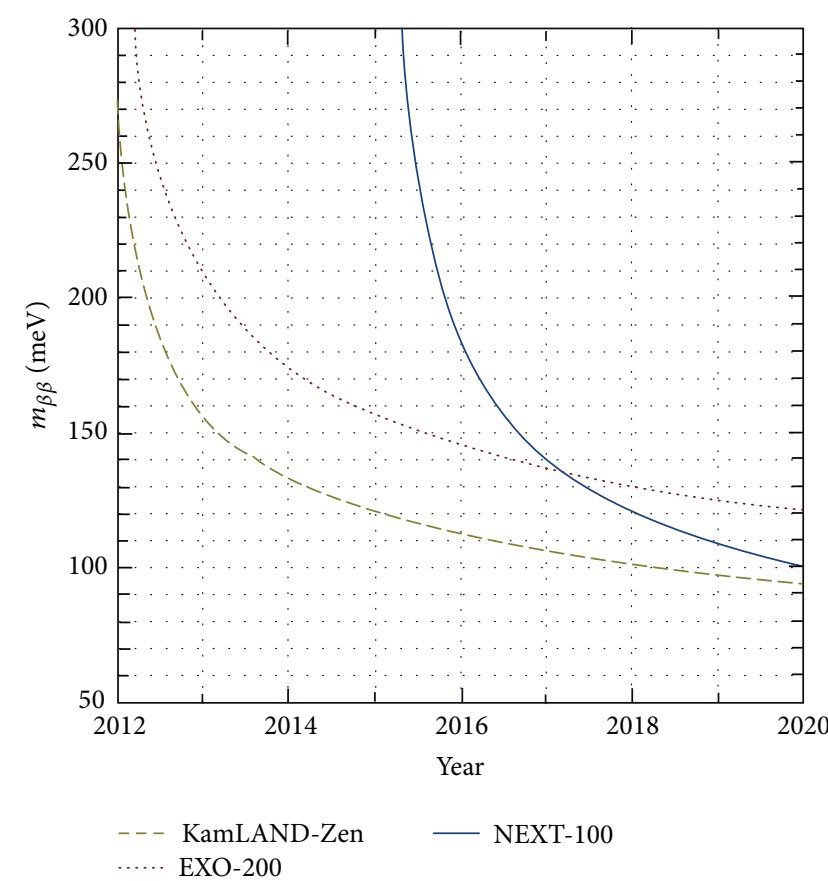

FIGURE 5: Sensitivity to the effective Majorana neutrino mass of the three xenon experiments as a function of the running time, assuming the parameters described in Table 1 . We consider a run of 8 years for EXO-200 and KamLAND-Zen (2012 to 2020) and a run of 5 years for NEXT (2015 to 2020).

measurements of the cosmic microwave background (CMB) $[3,4]$ :

$$
\sum m_{v} \equiv m_{1}+m_{2}+m_{3}<0.230 \mathrm{eV}(95 \% \mathrm{CL}),
$$

where $\sum m_{v}$ is the sum of the three neutrino masses $m_{1}, m_{2}$, and $m_{3}$.

Figure 4 shows the implications of such a measurement, when combined with the current limits from KamLANDZen and EXO. As it can be seen, the current sensitivity is not enough to explore significantly the inverse hierarchy, while Planck data exclude most of the so-called degenerate hierarchy. It follows that the next generation of $\beta \beta 0 \nu$ experiments must aim for extraordinary sensitivities to the effective neutrino mass. In particular, we will show that a sensitivity of $20 \mathrm{meV}$ in $m_{\beta \beta}$ is within the reach of a ton-scale HPXe detector. However, with luck, a discovery could be made before, if $m_{\beta \beta}$ is near $100 \mathrm{meV}$.

In order to gain a feeling of the potential of the NEXT technology it is interesting to compare the experimental parameters of the three xenon experiments, which are collected in Table 1. The parameters of EXO-200 and KamLAND-Zen are those published by the collaborations $[1,2]$. The resolution in NEXT corresponds to the most conservative result obtained by the NEXT prototypes [29], and the predicted background rate and efficiency come from the full background model of the collaboration [23,30], assuming a conservative background level for the dominant source of background (the energy-plane PMTs). Notice that the background rate of all the experiments is very good. The 
TABLE 1: Experimental parameters of the three xenon-based double beta decay experiments: (a) total mass of ${ }^{136}$ Xe, $M$; (b) $\beta \beta$ isotope enrichment fraction $f$, meaning the fraction of the mass enriched in the relevant isotope for $\beta \beta 0 v$; (c) total efficiency, $\varepsilon$, defined as the percentage of the total mass actually used as source times the signal detection efficiency, which depends on the fraction of signal that escapes detection; (d) energy resolution, $\delta E$, at the $Q$ value of ${ }^{136} \mathrm{Xe}$; and (e) background rate, $b$, in the region of interest around $Q_{\beta \beta}$ expressed in counts/(keV·kg.y) (shortened as ckky) [28].

\begin{tabular}{lccccc}
\hline Experiment & $M(\mathrm{~kg})$ & $f$ & $\varepsilon$ & $\delta E(\% \mathrm{FWHM})$ & 3.9 \\
EXO-200 & 110 & 0.81 & 52 & 9.9 & 1.5 \\
KamLAND-Zen & 330 & 0.91 & 62 & 0.7 & 1.0 \\
NEXT-100 & 100 & 0.91 & 30 & 0.5 \\
\hline
\end{tabular}

TABLE 2: Expected experimental parameters of the three xenonbased double beta decay technologies in a possible ton-scale experiment: (a) signal detection efficiency, which depends on the fraction of signal that escapes detection, $\varepsilon$; (b) energy resolution, $\delta E$, at the $Q$ value of ${ }^{136} \mathrm{Xe}$; and (c) background rate, $b$, in the region of interest around $Q_{\beta \beta}$ expressed in counts $/(\mathrm{keV} \cdot \mathrm{kg} \cdot \mathrm{y})$.

\begin{tabular}{lccc}
\hline Experiment & $\varepsilon$ & $\delta E(\%$ FWHM $)$ & $b\left(10^{-3}\right.$ ckky $)$ \\
\hline LXe & 38 & 3.2 & 0.1 \\
XeSci & 42 & 6.5 & 0.1 \\
HPXe & 30 & 0.5 & 0.1 \\
\hline
\end{tabular}

HPXe technology offers less efficiency than the other two but a much better resolution.

Figure 5 shows the expected performance of the three experiments, assuming the parameters described in Table 1 and the central value of the nuclear matrix elements described in [14]. The experimental sensitivity was computed following [14] and includes only statistical fluctuations. A full analysis including systematic errors would require a deeper knowledge of all the three experiments. We consider a run of five years for NEXT (2015 to 2020) and a longer run of eight years for EXO-200 and KamLAND-Zen (2012 to 2020). A total dead-time of $10 \%$ a year for all experiments is assumed. It follows that all three experiments will have a chance of making a discovery if $m_{\beta \beta}$ is in the range of $100 \mathrm{meV}$. The fact that the experiments are based on different experimental techniques, with different systematic errors, makes their simultaneous running even more attractive. The combination of the three can reach a sensitivity of about $65 \mathrm{meV}$ [28]. Notice that, in spite of its late start, NEXT sensitivity can surpass that of the other xenon experiments.

2.5. Towards the Ton Scale. To cover the full range allowed by the inverse hierarchy, one needs masses in the range of one ton of isotope. Xenon experiments have the potential to deploy those large masses (for instance, to enrich ${ }^{76} \mathrm{Ge}$ is 10 times more expensive than ${ }^{136} \mathrm{Xe}$ ). This characteristic, together with the fact that one can build large xenon-based TPCs or calorimeters, makes them a preferred choice for the next-to-next generation of experiments.

Table 2 summarises a projection [28] of the experimental parameters for the three technologies, while Figure 6 shows the expected performance of xenon experiments assuming the parameters described in Table 2, up to a total exposure of 10 ton'year. At the maximum exposure, the XeSci detectors

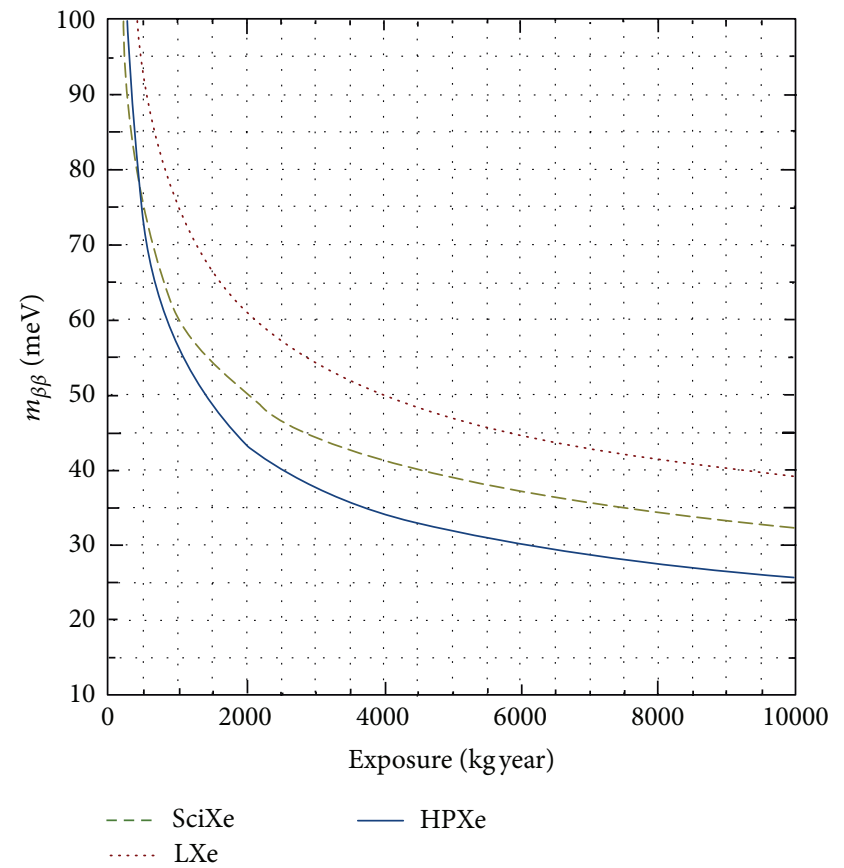

FIGURE 6: Sensitivity of the three technologies experiments as a function of the total exposure, assuming the parameters described in Table 2 [28].

reach a draw at $\sim 32 \mathrm{meV}$, while the HPXe detector reaches $25 \mathrm{meV}$. For further details, we refer the reader to [28].

To summarise, the NEXT experiment has an enormous interest for $\beta \beta 0 \nu$ searches not only due to its large physics potential-that is, the ability to discover that neutrinos are Majorana particles-but also as a springboard to the next-tonext generation of very challenging, ton-based experiments.

\section{The NEXT Detector}

3.1. The SOFT Concept. Xenon, as a detection medium, provides both scintillation and ionization as primary signals. To achieve optimal energy resolution, the ionization signal is amplified in NEXT using electroluminescence (EL). The electroluminescent light provides both a precise energy measurement and tracking. Following ideas introduced in [31] and further developed in our CDR [32], the chamber will have separated detection systems for tracking and calorimetry. This is the so-called SOFT concept, illustrated in Figure 7. 


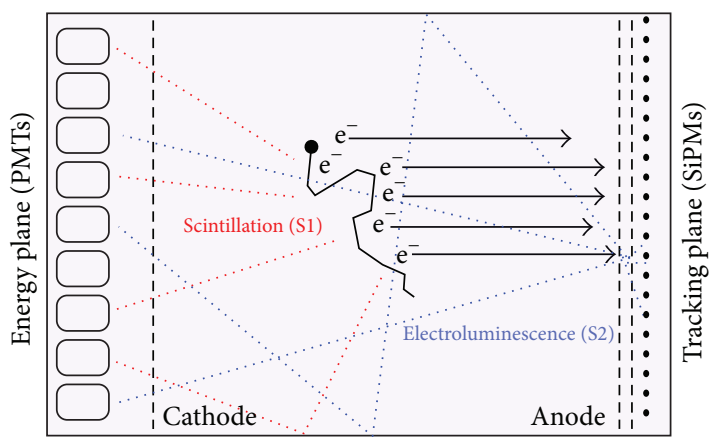

FIgure 7: The Separate, Optimized Functions (SOFT) concept in the NEXT experiment: EL light generated at the anode is recorded in the photosensor plane right behind it and used for tracking; it is also recorded in the photosensor plane behind the transparent cathode and used for a precise energy measurement.

The detection process is as follows. Particles interacting in the HPXe transfer their energy to the medium through ionization and excitation. The excitation energy is manifested in the prompt emission of VUV $(\sim 178 \mathrm{~nm})$ scintillation light (S1). The ionization tracks (positive ions and free electrons) left behind by the particle are prevented from recombination by an electric field $(0.03-0.05 \mathrm{kV} / \mathrm{cm} / \mathrm{bar})$. The ionization electrons drift toward the TPC anode (right part of the figure), entering a region, defined by two highly transparent meshes, with an even more intense electric field $(3 \mathrm{kV} / \mathrm{cm} / \mathrm{bar})$. There, further VUV photons are generated isotropically by electroluminescence (S2). Therefore, both scintillation and ionization produce an optical signal, to be detected with a sparse plane of PMTs (the energy plane) located behind the cathode. The detection of the primary scintillation light constitutes the start-of-event, whereas the detection of the EL light provides an energy measurement. Electroluminescent light provides tracking as well, since it is detected also a few millimeters away from production at the anode plane, via an array of $1 \mathrm{~mm}^{2} \mathrm{SiPMs}, 1 \mathrm{~cm}$ spaced (the tracking plane).

3.2. The Apparatus. Figure 8 shows a drawing of the NEXT100 detector, indicating all the major subsystems. These are as follows.

(i) The pressure vessel, built with stainless steel and designed to withstand a pressure of 15 bar, described in Section 3.2.1. A copper layer on the inside shields the sensitive volume from the radiation which originated in the vessel material.

(ii) The field cage, electrode grids, HV penetrators, and light tube, described in Section 3.2.2.

(iii) The energy plane, made of PMTs housed in copper enclosures, described in Section 3.2.3.

(iv) The tracking plane, made of SiPMs arranged into dice boards (DBs), described in Section 3.2.4.

(v) The gas system, capable of pressurizing, circulating, and purifying the gas, described in Section 3.2.5.

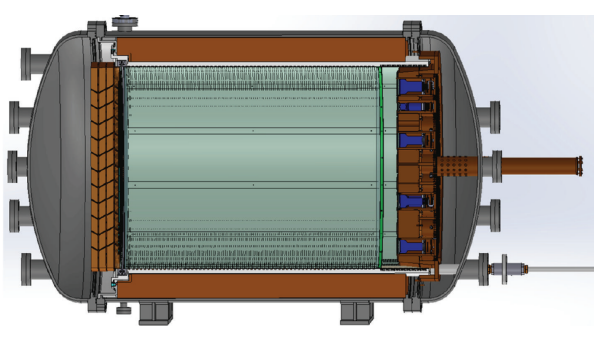

Figure 8: A 3D drawing of the NEXT-100 detector, showing the pressure vessel (gray), the internal copper shield (brown), and the field cage (green). The PMTs of the energy plane are shown in blue.

(vi) The front-end electronics, placed outside the chamber, described in Section 3.2.6.

(vii) Shielding and other infrastructures, described in Section 3.2.7.

The NEXT TDR [23] gives the details of the design and components of the detector, which we summarise briefly here.

3.2.1. The Pressure Vessel. The pressure vessel, shown in Figure 9, consists of a barrel central section with two identical torispheric heads on each end, their main flanges bolted together, and is made of stainless steel, specifically the lowactivity 316 Ti alloy. In order to shield the activity of the vessel, we introduce an inner copper shield, $12 \mathrm{~cm}$ thick and made of radiopure copper. It will attenuate the radiation coming from the external detector (including the vessel and the external lead shield) by a factor of 100 .

The vessel has been built strictly to ASME Pressure Vessel Design Code, Section VIII, by the Madrid-based company MOVESA. It has been designed almost entirely by the collaboration, under the leadership of the Lawrence Berkeley National Laboratory (LBNL) and the Instituto de Física Corpuscular (IFIC) in València. IFIC is in charge of supervision of fabrication, testing, certification, and transport to LSC.

3.2.2. The Field Cage. The main body of the field cage will be a high-density polyethylene (HDPE) cylindrical shell, $2.5 \mathrm{~cm}$ thick, which will provide electric insulation from the vessel. Three wire meshes separate the two electric field regions of the detector. The drift region, between the cathode and the first mesh of the electroluminescence region, is a cylinder of $107 \mathrm{~cm}$ of diameter and $130 \mathrm{~cm}$ of length. Copper strips are attached to the HDPE and are connected with low background resistors to the high voltage, in order to keep the field uniform across the drift region. The electroluminescence region is $1.0 \mathrm{~cm}$ long.

All the components of the field cage have been prototyped with the NEXT-DEMO detector (see Section 5.1). The NEXT100 field cage and ancillary systems will be built by our USA collaborators.

3.2.3. The Energy Plane. The energy measurement in NEXT is provided by the detection of the electroluminescence light by an array of photomultipliers, the energy plane, located behind the transparent cathode (Figure 10). Those PMTs will also 


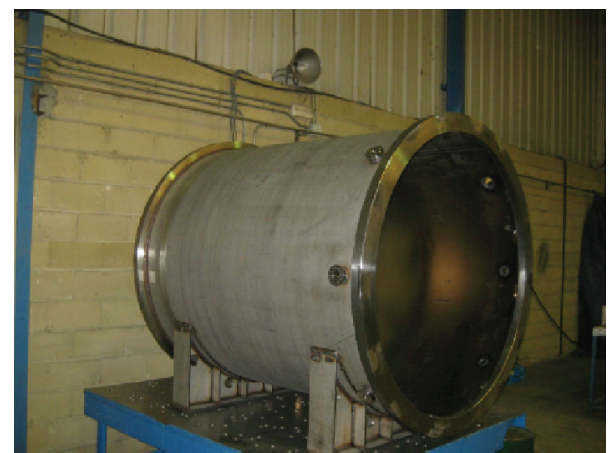

(a)

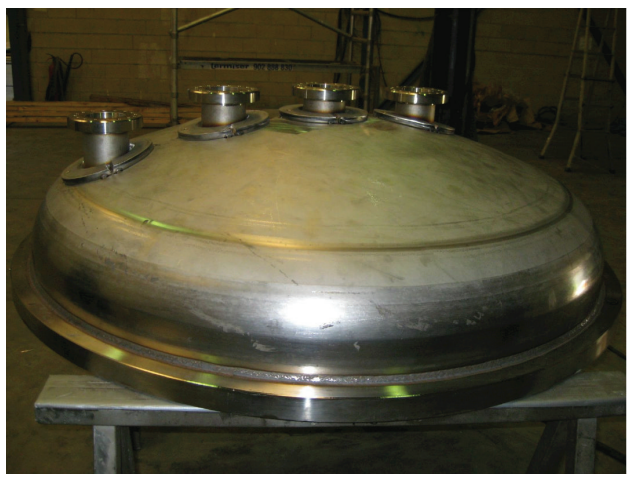

(b)

FIgURE 9: NEXT-100 vessel in the final stages of the production. Main body of the vessel (a) and endcap with the port to extract the different signals and circulate the gas (b).

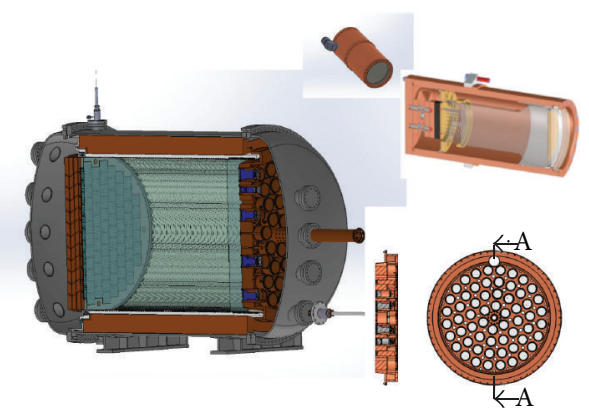

FIgURE 10: A drawing of the detector showing the energy plane inside the pressure vessel.

record the scintillation light that indicates the start of the event.

A total of 60 Hamamatsu R11410-10 photomultipliers (Figure 11) covering $32.5 \%$ of the cathode area constitute the energy plane. This 3-inch phototube model has been specially developed for radiopure, xenon-based detectors. The quantum efficiency of the R11410-10 model is around 35\% in the VUV and $30 \%$ in the blue region of the spectrum, and the dark count rate is $2-3 \mathrm{kHz}(0.3$ photoelectron threshold) at room temperature [33].

Pressure-resistance tests run by the manufacturer showed that the R11410-10 cannot withstand pressures above 6 atmospheres. Therefore, in NEXT-100 they will be sealed into individual pressure-resistant, vacuum-tight copper enclosures

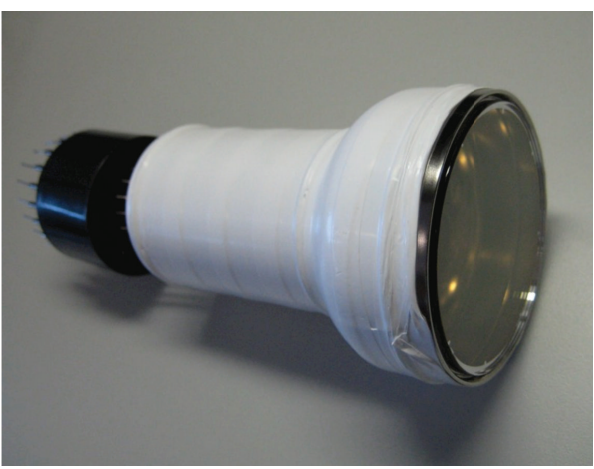

FIgURE 11: The Hamamatsu R11410-10.

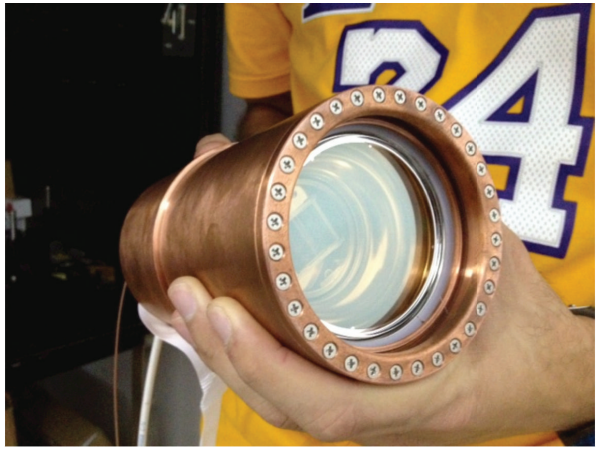

FIGURE 12: The pressure-resistant enclosure or "can" protecting the PMTs inside the pressure vessel.

coupled to sapphire windows (see Figure 12). The window, $5 \mathrm{~mm}$ thick, is secured with a screw-down ring and sealed with an O-ring to the front-end of the enclosure. A similar backcap of copper seals the back side of the enclosures. The PMT must be optically coupled to the window. The specific optical pad or gel to be used for this purpose is still being investigated. A spring on the backside pushes the photomultiplier against the optical pads. Since UV light is absorbed by sapphire more than blue light, the sapphire windows will be coated with a wavelength shifter material, in order to shift the UV light of the scintillation of xenon to blue wavelengths.

These PMT modules are all mounted to a common carrier plate that attaches to an internal flange of the pressure vessel head (see Figure 13). The enclosures are all connected via individual pressure-resistant, vacuum-tight tubing conduits to a central manifold and maintained at vacuum well below the Paschen minimum, avoiding sparks and glow discharge across PMT pins. The PMT cables are routed through the conduits and the central manifold to a feedthrough in the pressure vessel nozzle.

The design of the energy plane has been shared between the IFIC, the UPV (Universidad Politécnica de València), and the LBNL groups. The PMTs have already been purchased and tested and are currently being screened for radioactivity at the LSC. Prototype PMT enclosures have been built and a full prototype energy plane including 14 PMTs is under construction and will be tested at LSC in 2013. The full energy plane will be installed in the detector during 2014 . 


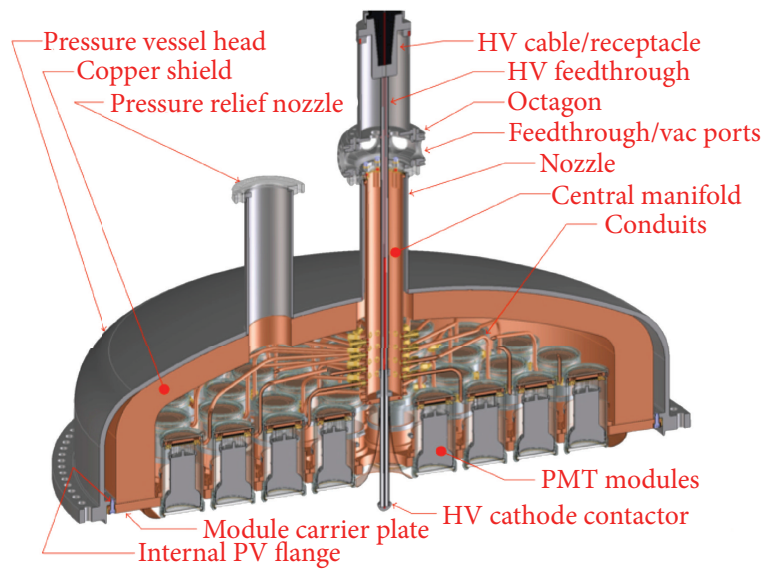

FIgURE 13: The full energy plane of NEXT-100 mounted in the vessel head.

3.2.4. The Tracking Plane. The tracking function in NEXT100 will be provided by a plane of silicon photomultipliers operating as sensor pixels and located behind the transparent EL gap. The chosen SiPMs are the S10362-11-050P model by Hamamatsu. This device has an active area of $1 \mathrm{~mm}^{2}$, 400 sensitive cells ( $50 \mu \mathrm{m}$ size), and high photon detection efficiency in the blue region (about $\sim 50 \%$ at $440 \mathrm{~nm}$ ). For this reason, they need to be coated with the same wavelength shifter used for the windows of the copper cans. SiPMs are very cost effective and their radioactivity is very low, given their composition (mostly silicon) and very small mass.

The SiPMs will be mounted in dice boards (DBs), identical to those prototyped in NEXT-DEMO (see Section 5.1). The electronics for the SiPMs will also be an improved version of the electronics for the DEMO detector. Also, like in NEXTDEMO, all the electronics will be outside the chamber. The large number of channels in NEXT-100, on the other hand, requires the design and fabrication of large custom-made feedthroughs to extract the signals.

In order to cover the whole field cage cross section with a pitch between sensors of $1 \mathrm{~cm}$, we need $\sim 8000$ SiPMs. The design of the DBs and the front-end electronics have been made at IFIC and UPV. The DBs have been fully tested in NEXT-DEMO and are ready for production.

A prototype of the LCFT will be tested in 2013. The full tracking plane can be installed in 2014.

3.2.5. The Gas System. The gas system, shown in Figure 14, must be capable of pressurizing, circulating, purifying, and depressurizing the NEXT-100 detector with xenon, argon, and possibly other gases with negligible loss and without damage to the detector. In particular, the probability of any substantial loss of the very expensive enriched xenon (EXe) must be minimized. A list of requirements, in approximate decreasing order of importance, considered during the design is given below.

(1) Pressurize vessel, from vacuum to 15 bar (absolute).

(2) Depressurize vessel to closed reclamation system, 15 bar to 1 bar (absolute), on fault, in 10 seconds maximum.
(3) Depressurize vessel to closed reclamation system, 15 bar to 1 bar (absolute), in normal operation, in 1 hour maximum.

(4) Relieve pressure (vent to closed reclamation system) for fire or other emergency condition.

(5) Allow a maximum leakage of EXe through seals (total combined) of $100 \mathrm{~g} /$ year.

(6) Allow a maximum loss of EXe to atmosphere of $10 \mathrm{~g} /$ year.

(7) Accommodate a range of gasses, including Ar and $\mathrm{N}_{2}$.

(8) Circulate all gasses through the detector at a maximum rate of 200 standard liters per minute (slpm) in axial flow pattern.

(9) Purify EXe continuously. Purity requirements: $<1 \mathrm{ppb}$ $\mathrm{O}_{2}, \mathrm{CO}_{2}, \mathrm{~N}_{2}$, and $\mathrm{CH}_{4}$.

The most vulnerable component of the gas system is the recirculation compressor, which must have sufficient redundancy to minimize the probability of failure and leakage. The compressor chosen is made with metal-to-metal seals on all the wetted surfaces. The gas is moved through the system by a triple stainless steel diaphragm. Between each of the diaphragms there is a sniffer port to monitor gas leakages. In the event of a leakage, automatic emergency shutdown can be initiated.

The gas system will be equipped with both roomtemperature and heated getters by SAES Pure Gas which remove electronegative impurities $\left(\mathrm{O}_{2}, \mathrm{H}_{2} \mathrm{O}\right.$, etc. $)$ from the xenon.

An automatic recovery system of the expensive EXe will also be needed to evacuate the chamber in case of an emergency condition. A $35 \mathrm{~m}^{3}$ expansion tank will be placed inside the laboratory to quickly reduce the gas pressure in the system. Additionally, for controlled gas evacuation, we will cryopump EXe into a liquid nitrogen cooled vessel.

The gas system has been designed as a collaboration between IFIC and the University of Zaragoza, taking advantage of the experience gained with our prototypes. The basic gas system needed for the initial operation of the NEXT-100 apparatus has already been purchased and shipped to LSC, but the system must be upgraded during 2014 for the enriched xenon run in 2015.

3.2.6. Electronics. The NEXT-100 data-acquisition system (DAQ), shown in Figure 15, follows a modular architecture named the Scalable Readout System (SRS), already described in our CDR [32]. At the top of the hierarchy, a PC farm running the DAQ software, DATE, receives event data from the DAQ modules via Gigabit Ethernet links. The DATE PCs (local data concentrators, LDCs) assemble incoming fragments into subevents, which are sent to one or more additional PCs (global data concentrators, GDCs). The GDCs build complete events and store them on disk for offline analysis.

The DAQ modules used are front-end concentrator (FEC) cards, which serve as the generic interface between the DAQ system and application-specific front-end modules. The FEC 


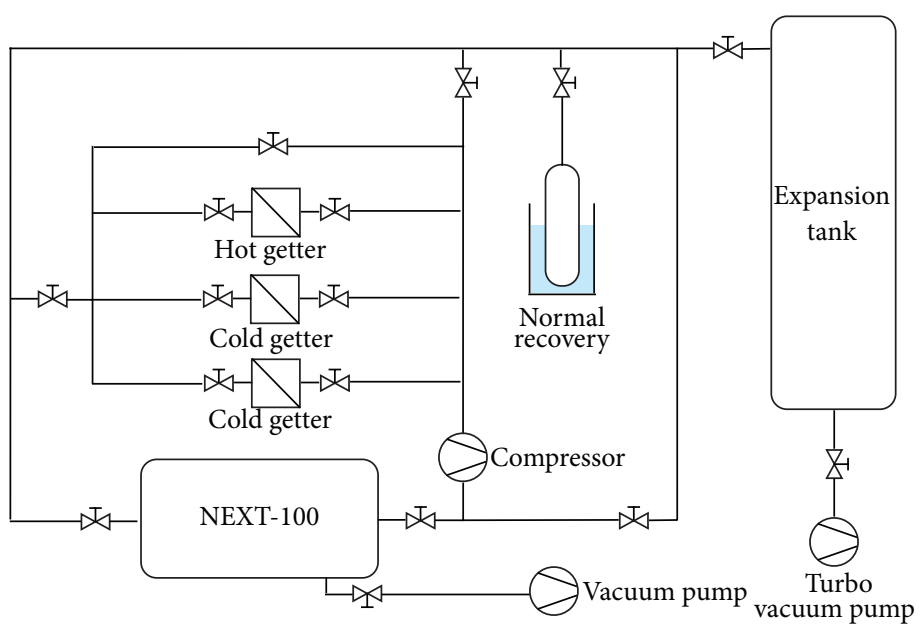

FIGURE 14: Scheme for the gas system of NEXT-100.

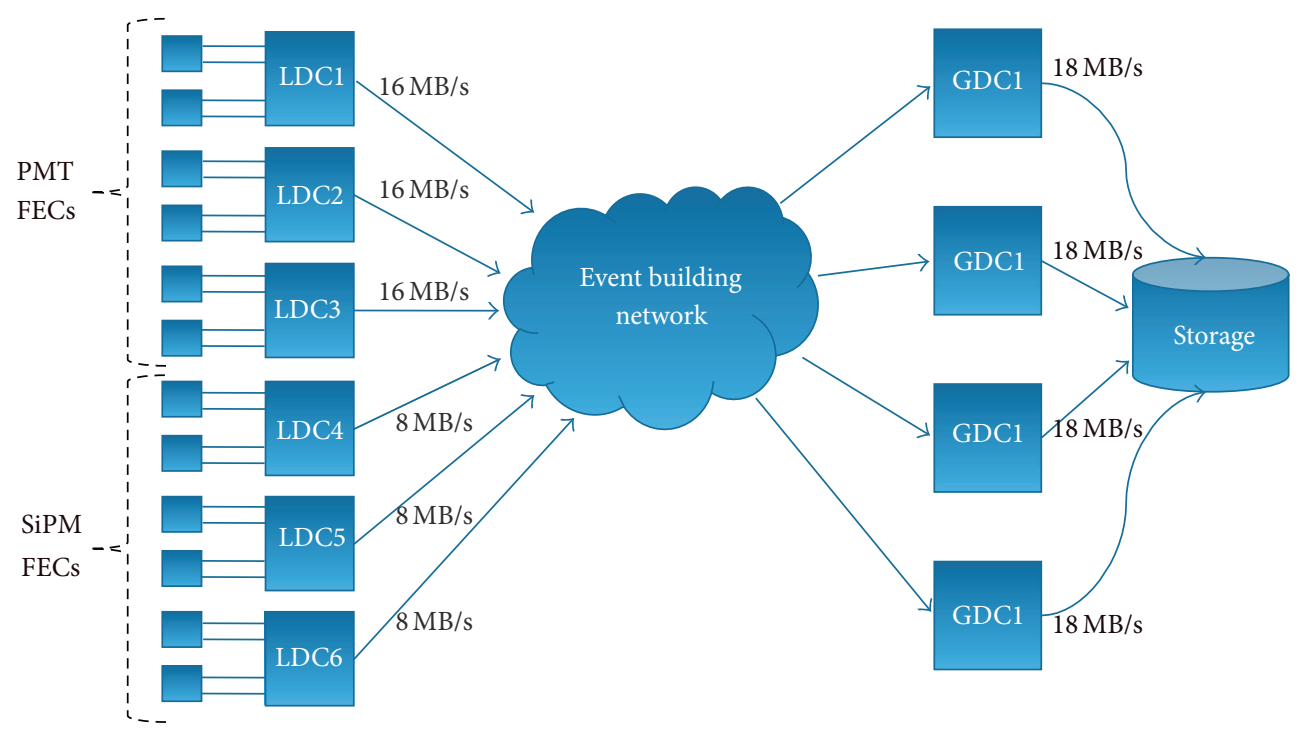

FIGURE 15: Scheme of the DAQ of NEXT-100.

module can interface different kinds of front-end electronics by using the appropriate plug-in card. The FEC card and the overall SRS concept have been developed within the framework of the CERN RD-51 collaboration [34]. Three different FEC plug-in cards are used in NEXT-100 (energy plane readout digitization, trigger generation, and tracking plane readout digitization).

Electronics for the Energy Plane. The front-end electronics for the PMTs in NEXT-100, shown in Figure 16, will be very similar to the system developed for the NEXT-DEMO and NEXT-DBDM prototypes. The first step in the chain is to shape and filter the fast signals produced by the PMTs (less than $5 \mathrm{~ns}$ wide) to match the digitizer and eliminate the high frequency noise. An integrator is implemented by simply adding a capacitor and a resistor to the PMT base. The charge integration capacitor shunting the anode stretches the pulse and reduces the primary signal peak voltage accordingly.
Our design uses a single amplification stage based on a fully differential amplifier, which features low noise $(2 \mathrm{nV} /$ $\sqrt{\mathrm{Hz}}$ ) and provides enough gain to compensate for the attenuation in the following stage, based on a passive RC filter with a cut frequency of $800 \mathrm{kHz}$. This filtering produces enough signal stretching to allow acquiring many samples per single photoelectron at $40 \mathrm{MHz}$, in the first stage of the DAQ.

Electronics for the Tracking Plane. The tracking plane will have $\sim 8000$ channels. On the other hand, the electronics for the SiPMs is simplified given the large gain of these devices. Our design consists of a very simple, 64-channel front-end board (Figure 17). Each board takes the input of a single DB (transmitted via low-crosstalk Kapton flat cables) and includes the analog stages, ADC converters, voltage regulators, and an FPGA that handles, formats, buffers, and transmits data to the DAQ. LVDS clock and trigger inputs 


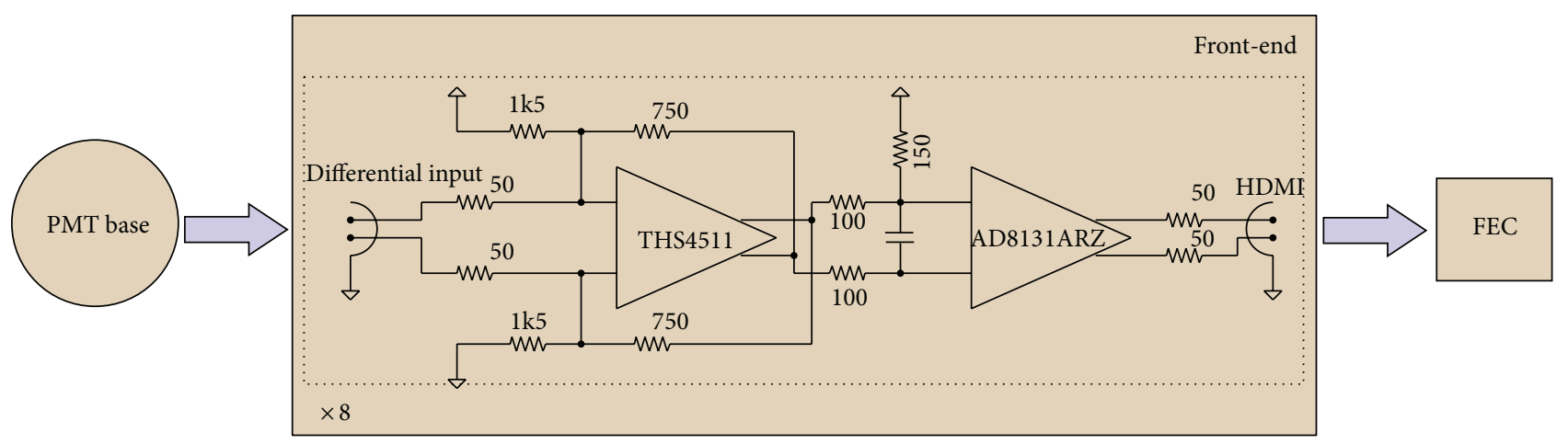

FIGURE 16: Scheme of the front-end for the energy plane.

are also needed. A total of 110 boards are required. The architecture of the boards is described in our TDR [23].

The design of the electronics is a collaboration between UPV and LBNL. It will be an evolution of the electronics currently operational at NEXT-DEMO. The DAQ is the responsibility of UPV, and it will also be an improved version of the DEMO DAQ.

3.2.7. Shielding and Other Infrastructures. To shield NEXT100 from the external flux of high energy gamma rays a relatively simple lead castle, shown in Figure 18, has been chosen, mostly due to its simplicity and cost-effectiveness. The lead wall has a thickness of $20 \mathrm{~cm}$ and is made of layers of staggered lead bricks held with a steel structure. The lead bricks have standard dimensions $\left(200 \times 100 \times 50 \mathrm{~mm}^{3}\right)$, and, by requirement, an activity in uranium and thorium lower than $0.4 \mathrm{mBq} / \mathrm{kg}$.

The lead castle is made of two halves mounted on a system of wheels that move on rails with the help of an electric engine. The movable castle has an open and a closed position. The former is used for the installation and service of the pressure vessel; the latter position is used in normal operation. A lock system fixes the castle to the floor in any of the two configurations to avoid accidental displacements.

The design of the lead castle has been led by the University of Girona, in collaboration with UPV and IFIC. The design is completed and the shield is ready to be built pending the availability of funds.

The construction of the infrastructures needed for the NEXT-100 experiment (working platform, seismic pedestal) is currently underway. They will be fully installed at LSC by the end of 2013.

Figure 19 shows an image of Hall A, future location of NEXT-100. The pool-like structure is intended to be a catchment reservoir to hold xenon or argon-a liquid-argon experiment, ArDM, will be neighbouring NEXT-100 in Hall A-gas in the event of a catastrophic leak. Therefore, for reasons of safety all experiments must preclude any personnel working below the level of the top of the catchment reservoir.

An elevated working platform has already been built. It is designed to stand a uniform load of $1500 \mathrm{~kg} / \mathrm{m}^{2}$ and a concentrated load of $200 \mathrm{~kg} / \mathrm{m}^{2}$. It is anchored to the hall ground and walls. The platform floor tiles are made of galvanized steel and have standard dimensions to minimize cost.

Due to the mild seismic activity of the part of the Pyrenees where LSC is located, a comprehensive seismic study has been conducted as part of the project risk analysis. As a result, an antiseismic structure that will hold both the pressure vessel and the shielding has been designed. This structure will be anchored directly to the ground and independent of the working platform to allow seismic displacements in the event of an earthquake.

\section{NEXT Background Model}

The NEXT background model describes the sources of radioactive contaminants in the detector and their activity. It allows us, via detailed simulation, to predict the background events that can be misidentified as signal.

\subsection{Sources of Background}

4.1.1. Radioactive Contaminants in Detector Materials. After the decay of ${ }^{214} \mathrm{Bi}$, the daughter isotope, ${ }^{214} \mathrm{Po}$, emits a number of deexcitation gammas with energies above $2.3 \mathrm{MeV}$. The gamma line at $2447 \mathrm{keV}$, of intensity $1.57 \%$, is very close to the $Q$ value of ${ }^{136} \mathrm{Xe}$. The gamma lines above $Q_{\beta \beta}$ have low intensity and their contribution is negligible.

The daughter of ${ }^{208} \mathrm{Tl},{ }^{208} \mathrm{~Pb}$, emits a de-excitation photon of $2614 \mathrm{keV}$ with a $100 \%$ intensity. The Compton edge of this gamma is at $2382 \mathrm{keV}$, well below $Q_{\beta \beta}$. However, the scattered gamma can interact and produce other electron tracks close enough to the initial Compton electron so they are reconstructed as a single object falling in the energy ROI. Photoelectric electrons are produced above the ROI but can lose energy via bremsstrahlung and populate the window, in case the emitted photons escape out of the detector. Paircreation events are not able to produce single-track events in the ROI.

4.1.2. Radon. Radon constitutes a dangerous source of background due to the radioactive isotopes ${ }^{222} \mathrm{Rn}$ (half-life of $3.8 \mathrm{~d}$ ) from the ${ }^{238} \mathrm{U}$ chain and ${ }^{220} \mathrm{Rn}$ (half-life of $55 \mathrm{~s}$ ) from 


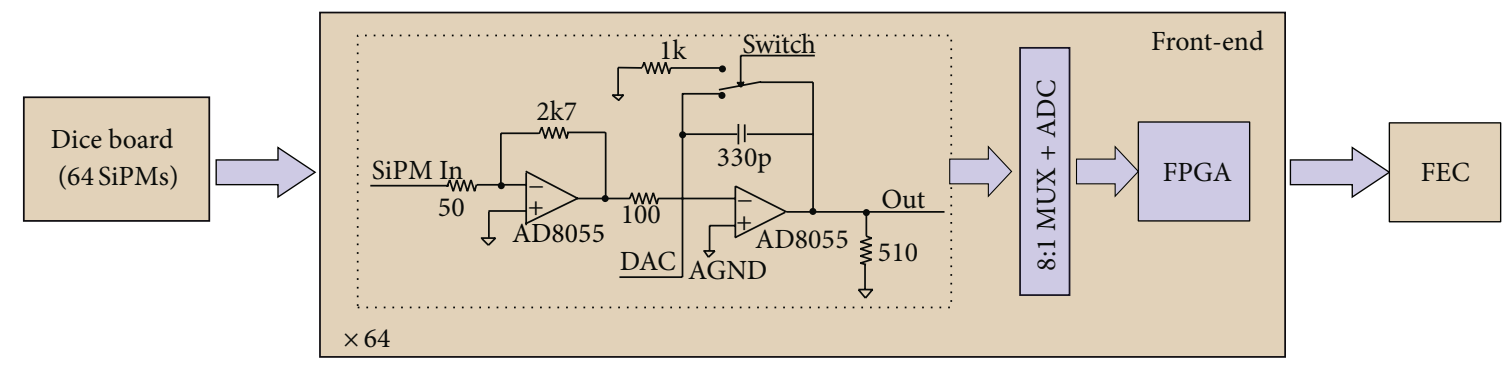

FIGURE 17: Scheme of the front-end for the tracking plane.

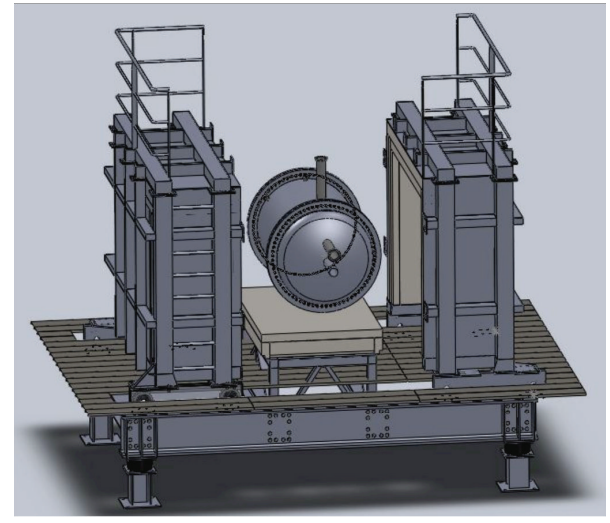

Figure 18: Drawing of the NEXT-100 lead castle shield in its open configuration.

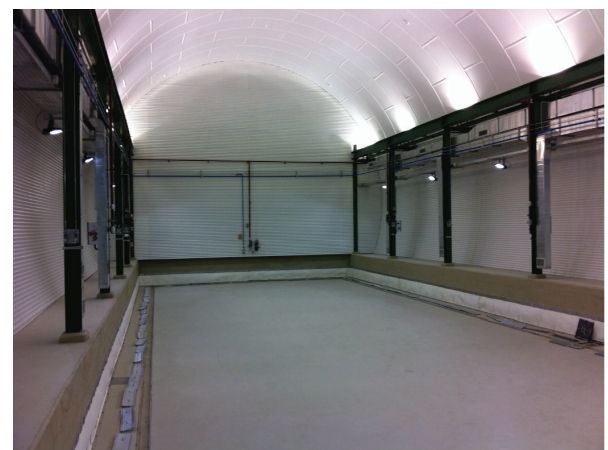

Figure 19: View of Hall A of the Laboratorio Subterráneo de Canfranc prior to any equipment installation.

the ${ }^{232} \mathrm{Th}$ chain. As a gas, it diffuses into the air and can enter the detector. ${ }^{214} \mathrm{Bi}$ is a decay product of ${ }^{222} \mathrm{Rn}$ and ${ }^{208} \mathrm{Tl}$ a decay product of ${ }^{220} \mathrm{Rn}$. In both cases, radon undergoes an alpha decay into polonium, producing a positively charged ion which is drifted towards the cathode by the electric field of the TPC. As a consequence, ${ }^{214} \mathrm{Bi}$ and ${ }^{208} \mathrm{Tl}$ contaminations can be assumed to be deposited on the cathode surface. Radon may be eliminated from the TPC gas mixture by recirculation through appropriate filters. In order to suppress radon in the volume defined by the shielding, it is possible to use continuously flowing nitrogen inside a bag that occupies the whole space left between the TPC and the shielding.
Radon control is a major task for a $\beta \beta 0 \nu$ experiment and will be of uppermost importance for NEXT-100.

4.1.3. Cosmic Rays and Laboratory Rock Backgrounds. Cosmic particles can also affect our experiment by producing high energy photons or activating materials. This is the reason why double beta decay experiments are conducted deep underground. At these depths, muons are the only surviving cosmic ray particles, but their interactions with the rock produce neutrons and electromagnetic showers. Furthermore, the rock of the laboratory itself is a rather intense source of ${ }^{208} \mathrm{Tl}$ and ${ }^{214} \mathrm{Bi}$ backgrounds as well as neutrons.

The flux of photons emanating from the LSC walls is (see our TDR and references therein [23])

(i) $0.71 \pm 0.12 \gamma / \mathrm{cm}^{2} / \mathrm{s}$ from the ${ }^{238} \mathrm{U}$ chain,

(ii) $0.85 \pm 0.07 \gamma / \mathrm{cm}^{2} / \mathrm{s}$ from the ${ }^{232} \mathrm{Th}$ chain.

These measurements include all the emissions in each chain. The flux corresponding to the ${ }^{208} \mathrm{Tl}$ line at $2614.5 \mathrm{keV}$ and the flux corresponding to the ${ }^{214} \mathrm{Bi}$ line at $1764.5 \mathrm{keV}$ were also measured (from the latter it is possible to deduce the flux corresponding to the $2448 \mathrm{keV}$ line). The results are

(i) $0.13 \pm 0.01 \mathrm{\gamma} / \mathrm{cm}^{2} / \mathrm{s}$ from the ${ }^{208} \mathrm{Tl}$ line,

(ii) $0.006 \pm 0.001 \mathrm{\gamma} / \mathrm{cm}^{2} / \mathrm{s}$ from the ${ }^{214} \mathrm{Bi}$ line at $2448 \mathrm{keV}$.

The above backgrounds are considerably reduced by the shielding. In addition, given the topological capabilities of NEXT, the residual muon and neutron background do not appear to be significant for our experiment.

4.2. Radioactive Budget of NEXT-100. Information on the radiopurity of the materials expected to be used in the construction of NEXT-100 has been compiled, performing specific measurements and also examining data from the literature for materials not yet screened. A detailed description is presented in [35]. A brief summary of the results presented there for the main materials is shown in Table 3.

4.3. Expected Background Rate. The only relevant backgrounds for NEXT are the photons emitted by the ${ }^{208} \mathrm{Tl}$ line $(2614.5 \mathrm{keV})$ and the ${ }^{214} \mathrm{Bi}$ line $(2448 \mathrm{keV})$. These sit very near $Q_{\beta \beta}$ and the interaction of the photons in the gas can fake the $\beta \beta 0 \nu$ signal. NEXT-100 has the structure of a Matryoshka 
TABLE 3: Activity (in $\mathrm{mBq} / \mathrm{kg}$ ) of the most relevant materials used in NEXT.

\begin{tabular}{lcccc}
\hline Material & Subsystem & ${ }^{238} \mathrm{U}$ & ${ }^{232}$ Th & Reference \\
\hline Lead & Shielding & 0.37 & 0.07 & {$[35]$} \\
Copper & Inner copper shielding & $<0.012$ & $<0.004$ & {$[35]$} \\
Steel (316Ti ) & Vessel & $<0.57$ & $<0.54$ & {$[35]$} \\
Polyethylene & Field cage & 0.23 & $<0.14$ & {$[36]$} \\
PMT (R11410-MOD, per piece) & Energy plane & $<2.5$ & $<2.5$ & {$[36]$} \\
\hline
\end{tabular}

TABle 4: Acceptance of the selection cuts for signal and backgrounds.

\begin{tabular}{lccc}
\hline \multirow{2}{*}{ Selection cut } & \multicolumn{3}{c}{ Fraction of events } \\
& $\beta \beta 0 \nu$ & ${ }^{214} \mathrm{Bi}$ & ${ }^{208} \mathrm{Tl}$ \\
\hline Confined, single track & 0.48 & $6.0 \times 10^{-5}$ & $2.4 \times 10^{-3}$ \\
Energy ROI & 0.33 & $2.2 \times 10^{-6}$ & $1.9 \times 10^{-6}$ \\
Topology $\beta \beta 0 \nu$ & 0.25 & $1.9 \times 10^{-7}$ & $1.8 \times 10^{-7}$ \\
\hline
\end{tabular}

(a Russian nesting doll). The flux of gammas emanating from the LSC walls is drastically attenuated by the lead castle, and the residual flux, together with that emitted by the lead castle itself and the materials of the pressure vessel, is further attenuated by the inner copper shielding. One then needs to add the contributions of the "inner elements" in NEXT: field cage, energy plane, and the elements of the tracking plane not shielded by the ICS.

A detailed Geant4 [37] simulation of the NEXT-100 detector was written in order to compute the background rejection factor achievable with the detector. Simulated events, after reconstruction, were accepted as a $\beta \beta 0 \nu$ candidate if

(a) they were reconstructed as a single track confined within the active volume;

(b) their energy fell in the region of interest, defined as $\pm 0.5 \mathrm{FWHM}$ around $Q_{\beta \beta}$;

(c) the spatial pattern of energy deposition corresponded to that of a $\beta \beta 0 \nu$ track (blobs at both ends).

The achieved background rejection factor together with the selection efficiency for the signal is shown in Table 4. As can be seen, the cuts suppress the radioactive background by more than 7 orders of magnitude. This results in an estimated background rate of about $5 \times 10^{-4}$ counts/(keV·kg·y).

\section{The NEXT EL Prototypes}

To prove the innovative concepts behind the NEXT design we have built two EL prototypes.

(i) NEXT-DEMO, operating at IFIC. This is a large prototype, which can hold a mass similar to that of the Gotthard experiment. It is conceived to fully test and demonstrate the EL technology.

(ii) NEXT-DBDM, operating at LBNL. This was our first operative prototype and has demonstrated a superb resolution, which extrapolates to $0.5 \% \mathrm{FWHM}$ at $Q_{\beta \beta}$.
The two prototypes are fully operational since 2011 and our initial results and operation experience have recently been published $[29,38,39]$.

5.1. NEXT-DEMO. In this section we describe in more detail the NEXT-DEMO demonstrator and our first results. The main goal of the prototype was the demonstration of the detector concept to be used in NEXT-100, more specifically

(1) to demonstrate good energy resolution (better than $1 \% \mathrm{FWHM}$ at $Q_{\beta \beta}$ ) in a large system with full spatial corrections,

(2) to demonstrate track reconstruction and the performance of SiPMs.

(3) to test long drift lengths and high voltages,

(4) to understand gas recirculation in a large volume, including operation stability and robustness against leaks,

(5) to understand the light collection of the detector, with and without using a wavelength shifter.

The apparatus, shown in Figure 20, is a high-pressure xenon electroluminescent TPC implementing the SOFT concept. Its active volume is $30 \mathrm{~cm}$ long. A $5 \mathrm{~mm}$ thick tube of hexagonal cross section made of PTFE is inserted into the active volume to improve the light collection. The TPC is housed in a stainless-steel pressure vessel, $60 \mathrm{~cm}$ long and with a diameter of $30 \mathrm{~cm}$, which can withstand up to 15 bar. Natural xenon circulates in a closed loop through the vessel and a system of purifying filters. The detector is not radiopure and is not shielded against natural radioactivity. It is installed in an operation room (see Figure 21) at IFIC, in València, Spain.

The time projection chamber itself is shown in Figure 22. Three metallic wire grids-called cathode, gate, and anodedefine the two active regions: the $30 \mathrm{~cm}$ long drift region, between cathode and gate, and the $0.5 \mathrm{~cm}$ long EL region, between gate and anode. The electric field is created by supplying a large negative voltage to the cathode and then degrading it using a series of metallic rings of $30 \mathrm{~cm}$ in diameter $5 \mathrm{~mm}$ spaced and connected via $5 \mathrm{G} \Omega$ resistors. The gate is at negative voltage so that a moderate electric fieldtypically of 2.5 to $3 \mathrm{kVcm}^{-1} \mathrm{bar}^{-1}$-is created between the gate and the anode, which is at ground. A buffer region of $10 \mathrm{~cm}$ between the cathode and the energy plane protects the latter from the high voltage by degrading it safely to ground potential. 


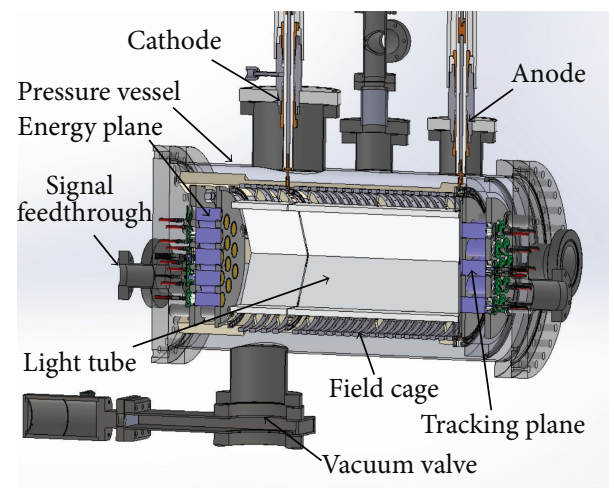

Figure 20: Cross section drawing of the NEXT-DEMO detector with all major parts labelled.

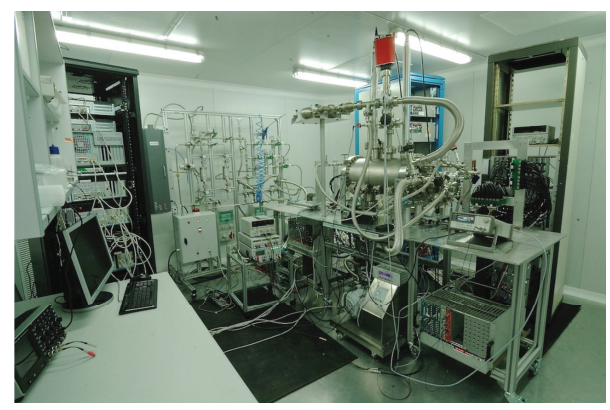

FIGURE 21: The NEXT-DEMO detector and ancillary systems (gas system, front-end electronics, and DAQ) in their location in the operation room at IFIC.

The high voltage is supplied to the cathode and the gate through custom-made high voltage feedthroughs (HVFT), shown in Figure 23, built pressing a stainless-steel rod into a Tefzel (a plastic with high dielectric strength) tube, which is then clamped using plastic ferrules to a CF flange. They have been tested to high vacuum and $100 \mathrm{kV}$ without leaking or sparking.

A set of six panels made of polytetrafluoroethylene (PTFE) are mounted inside the electric-field cage forming a light tube of hexagonal cross section (see Figure 24) with an apothem length of $8 \mathrm{~cm}$. PTFE is known to be an excellent reflector in a wide range of wavelengths [40], thus improving the light collection efficiency of the detector. In a second stage, the panels were vacuum-evaporated with tetraphenyl butadiene (TPB) - which shifts the UV light emitted by xenon to blue ( $430 \mathrm{~nm})$ - in order to study the improvement in reflectivity and light detection. Figure 24(b) shows the light tube illuminated with a UV lamp after the coating.

Six bars manufactured from PEEK, a low outgassing plastic, hold the electric-field cage and the energy plane together. The whole structure is attached to one of the endcaps using screws and introduced inside the vessel with the help of a rail system. All the TPC structures and the HVFT were designed and built by Texas A\&M.

The energy plane (see Figure 25) is equipped with 19 Hamamatsu R7378A photomultiplier tubes. These are 1 inch,

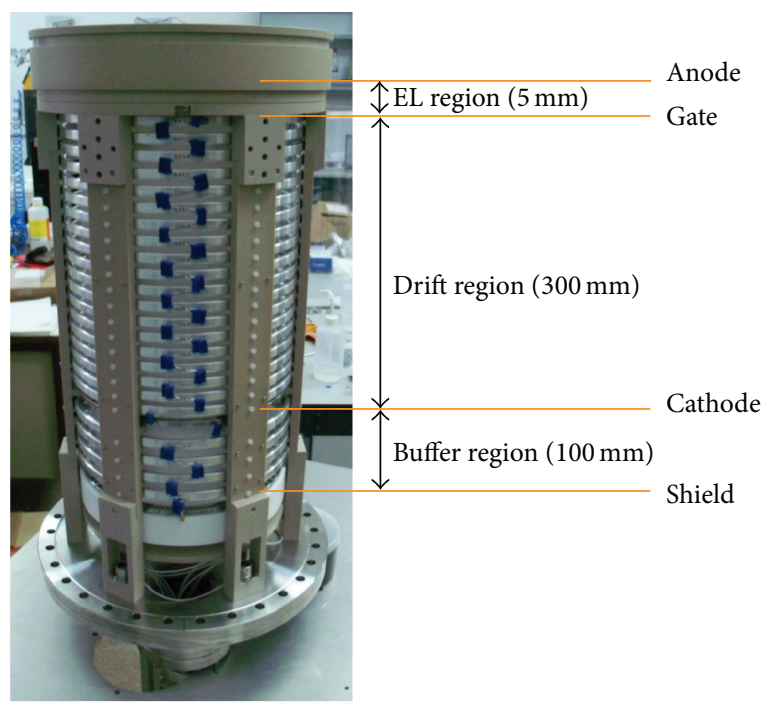

FIGURE 22: External view of the time projection chamber mounted on one endcap. The approximate positions of the different regions of the TPC are indicated.

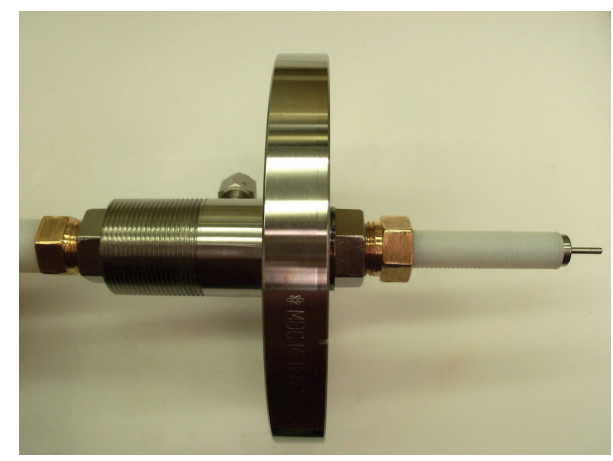

FIGURE 23: The NEXT-DEMO high-voltage feedthrough, designed and built by Texas A\&M.

pressure-resistant (up to 20 bar) PMTs with acceptable quantum efficiency $(\sim 15 \%)$ in the VUV region and higher efficiency at TPB wavelengths ( 25\%). The resulting photocathode coverage of the energy plane is about $39 \%$. The PMTs are inserted into a PTFE holder following a hexagonal pattern. A grid, known as shield and similar to the cathode but with the wires spaced $0.5 \mathrm{~cm}$ apart, is screwed on top of the holder and set to $\sim 500 \mathrm{~V}$. As explained above, this protects the PMTs from the high-voltage set in the cathode and ensures that the electric field in the $10 \mathrm{~cm}$ buffer region is below the EL threshold.

The initial operation of NEXT-DEMO implemented a tracking plane made of 19 pressure-resistant photomultipliers, identical to those used in the energy plane but operated at a lower gain. Instrumenting the tracking plane with PMTs during this period simplified the initial commissioning, debugging, and operation of the detector due to the smaller number of readout channels (19 PMTs in contrast to the $256 \mathrm{SiPMs}$ currently operating in the tracking plane) and their intrinsic sensitivity to the UV light emitted by xenon. 


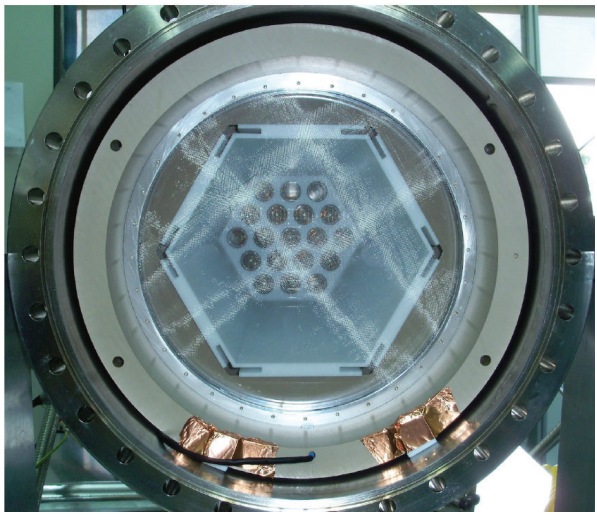

(a)

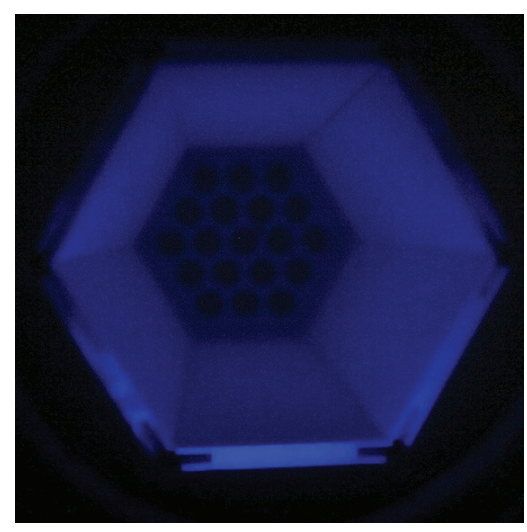

(b)

FIgURE 24: View of the light tube from the position of the tracking plane. (a) The meshes of the EL region can be seen in the foreground, and in the background, at the end of the light tube, the PMTs of the energy plane are visible. (b) The light tube of NEXT-DEMO illuminated with a UV lamp after being coated with TPB.

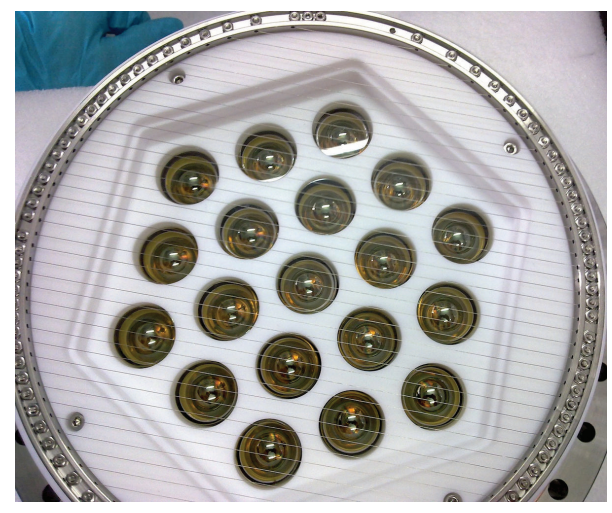

FIGURE 25: The energy plane of NEXT-DEMO, equipped with 19 Hamamatsu R7378A PMTs.

Since October 2012, NEXT-DEMO has been operating with a full tracking plane made with SiPMs, as shown in Figure 26. It consists of four boards, containing $8 \times 8 \mathrm{SiPMs}$ each, $1 \mathrm{~cm}$ spaced. Its higher granularity allows a finer position reconstruction in the plane orthogonal to the drift axis, thus increasing the fiducial volume of the chamber. SiPMs are not sensitive to VUV light, but they are to blue light, and therefore they had to be coated with TPB. The isotropical light emission of TPB, together with an improvement of the reflectivity of PTFE for wavelengths in the blue range, produced an overall increase of the collected light.

Figure 27 shows the measured energy spectrum of $511 \mathrm{keV}$ gamma rays from ${ }^{22} \mathrm{Na}$ in the fiducial volume of NEXTDEMO. A Gaussian fit to the photoelectric peak indicates an energy resolution of $1.82 \%$ FWHM. Extrapolating the result to the $Q$ value of ${ }^{136} \mathrm{Xe}(2458 \mathrm{keV})$ assuming a $E^{-1 / 2}$ dependence, we obtain a resolution of $0.83 \%$ FWHM, better than the NEXT target resolution of $1 \%$ FWHM at $Q_{\beta \beta}$. The DEMO apparatus measures electrons in a large fiducial volume, and therefore this result can be safely extrapolated to NEXT-100. We believe that an ultimate resolution of $0.5 \%$

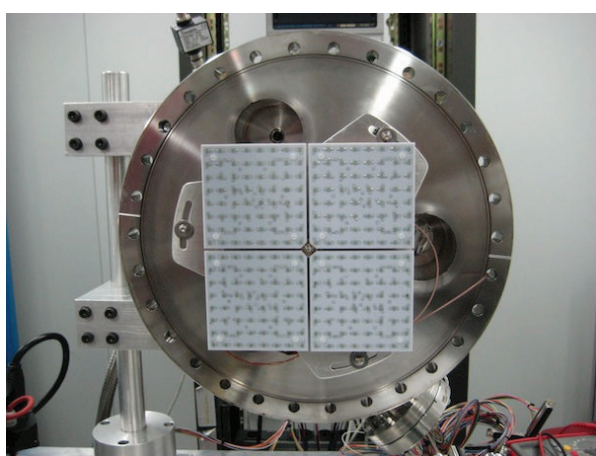

Figure 26: Dice boards installed in NEXT-DEMO, containing 64 $(8 \times 8)$ MPPCs each. There will be about 100 such boards in NEXT100.

FWHM, as found by DBDM (see the next section), can eventually be attained.

A first approximation of the event topology reconstruction is performed subdividing the charge in time slices $(z$ dimension) and reconstructing a single $x y$ point per slice. A further detailed analysis to allow the reconstruction of multiple depositions per slice is being studied. For this analysis, a slice width of $4 \mu \mathrm{s}$ is used as it gives enough information in the tracking plane to achieve a reliable $x y$ reconstruction and it is also comparable to the time an electron needs to cross the EL gap. The $x y$ position of a slice is reconstructed using the averaged position of the SiPMs with higher recorded secondary scintillation signal, weighted with their collected integrated charge. The energy associated with this position is recorded in the cathode for the same time interval, so that the $d E / d z$ of the event can be studied. The energy and position information are then used to calculate a cubic spline between the individual points in order to obtain a finer description of the path (see Figure 28).

The first reconstructed events (Figure 29) demonstrate the topology capabilities of the NEXT technology. The reconstructed electrons show a random walk into the gas 


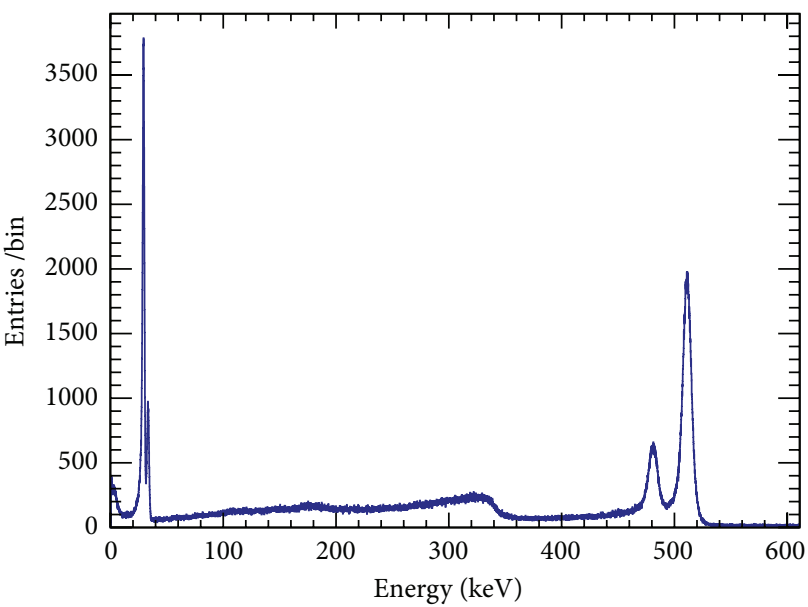

(a)

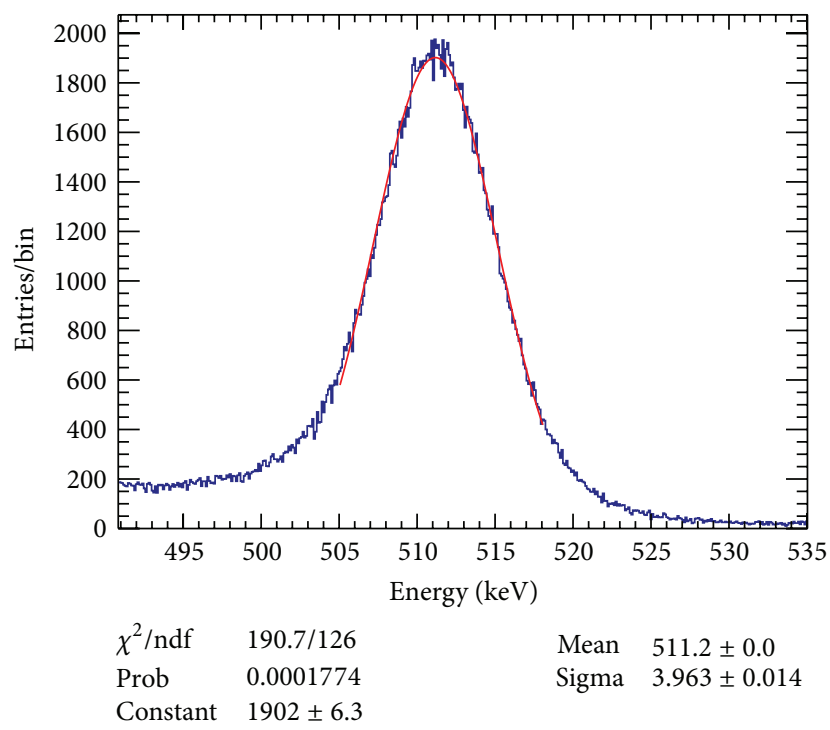

(b)

FIGURE 27: Energy spectra for ${ }^{22} \mathrm{Na}$ gamma-ray events within the fiducial volume of NEXT-DEMO. (a) The whole spectrum. (b) Zoom-in the photoelectric peak [41].

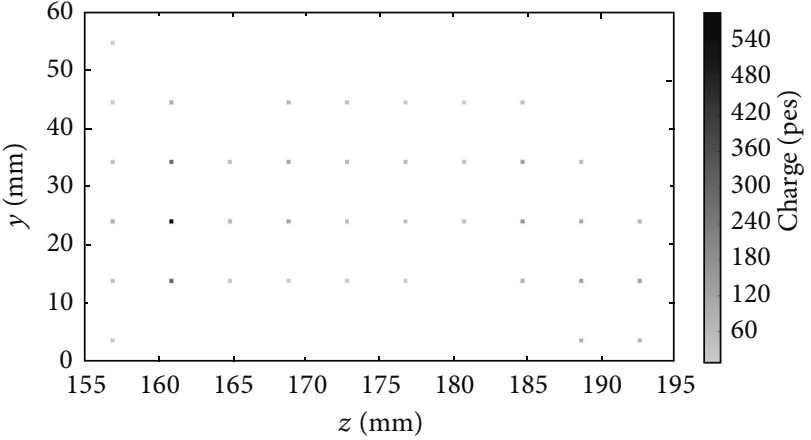

(a)

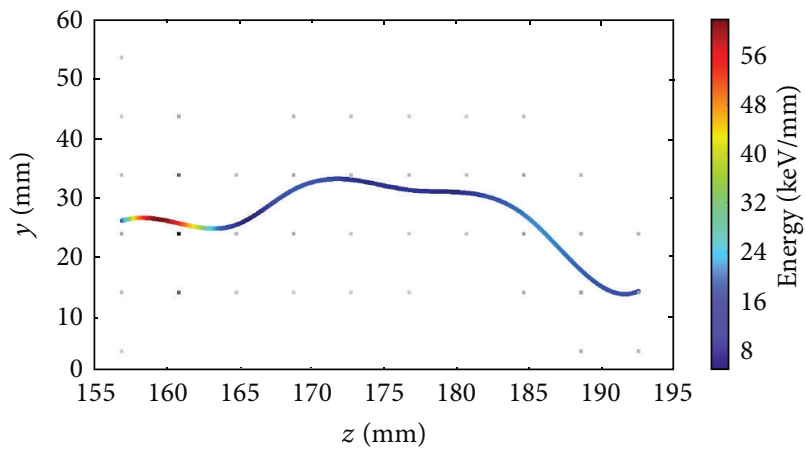

(c)

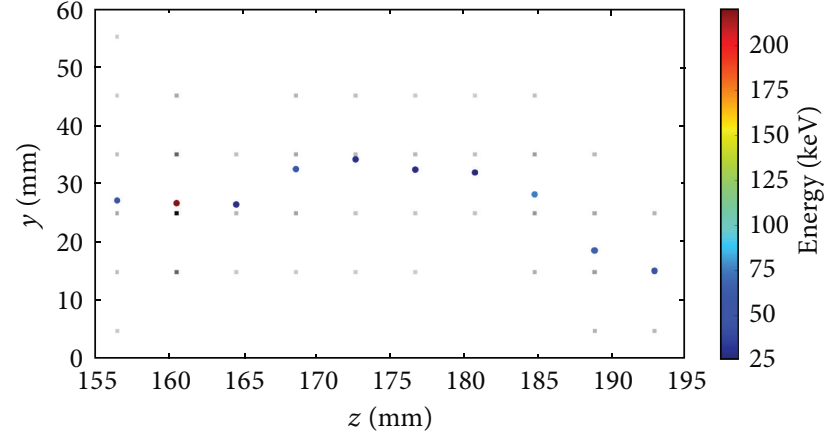

(b)

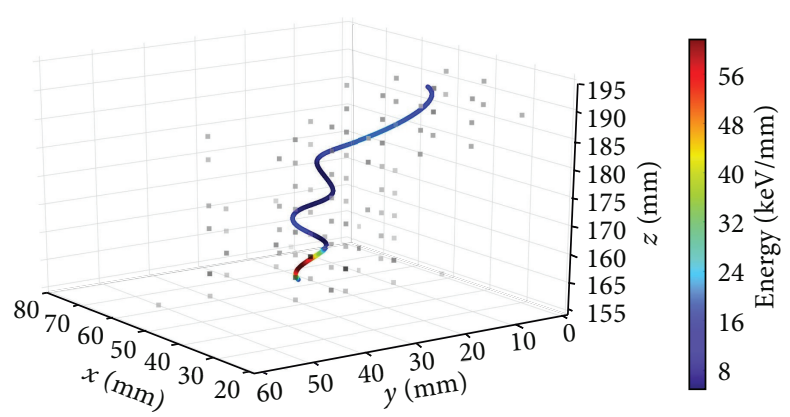

(d)

Figure 28: Example of the reconstruction of a ${ }^{137} \mathrm{Cs}$ track. The charge of the different SiPMs is split into slices of $4 \mathrm{~mm}$ in width in $z$ (a). One point is calculated for each slice using the barycentre method and the energy of the points is then associated with the measurement made in the cathode (b). A cubic spline is used to interconnect the different points. The result is shown in the bottom line: $Y Z$ projection (c) and 3D image (d) of the reconstructed track [41], where the $z$ coordinate is the axis of the cylindrical TPC and $x y$ define the orthogonal plane. 


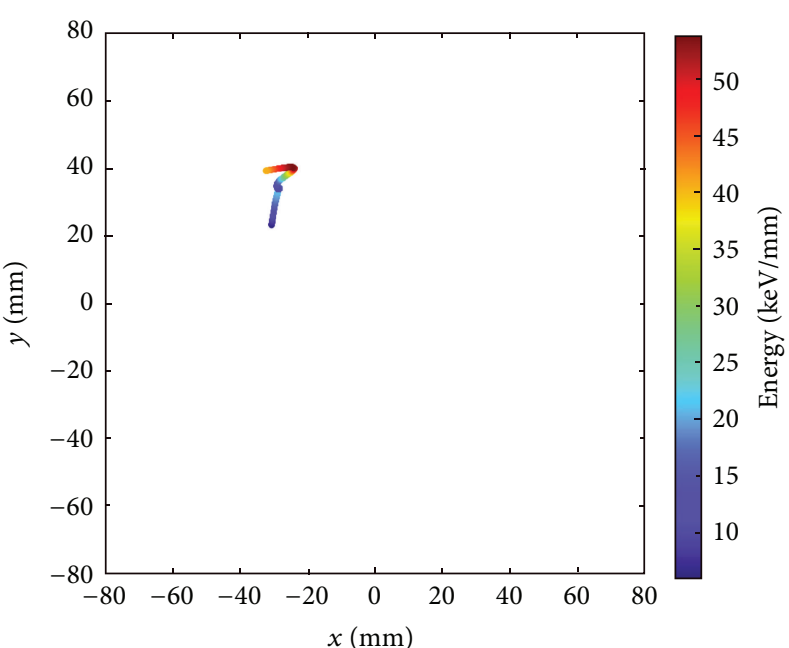

(a)

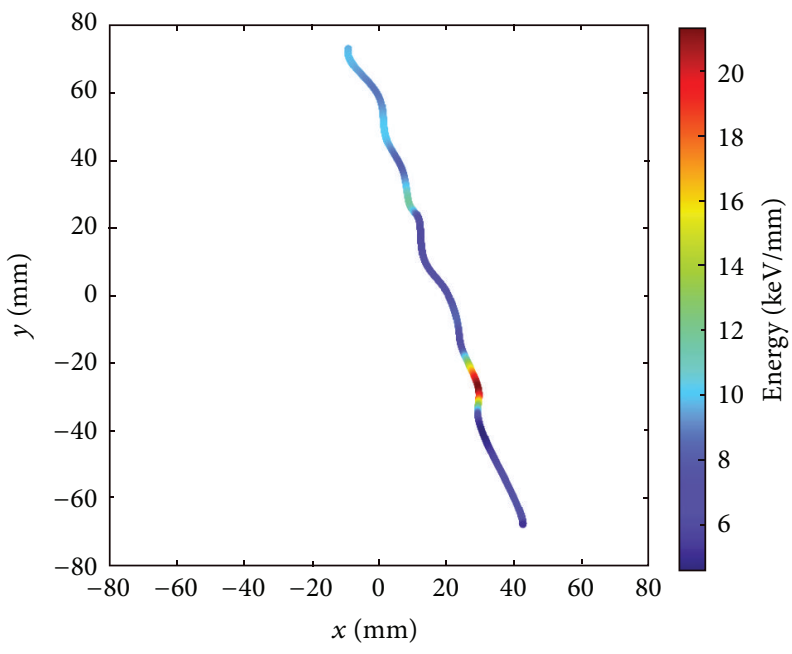

(c)

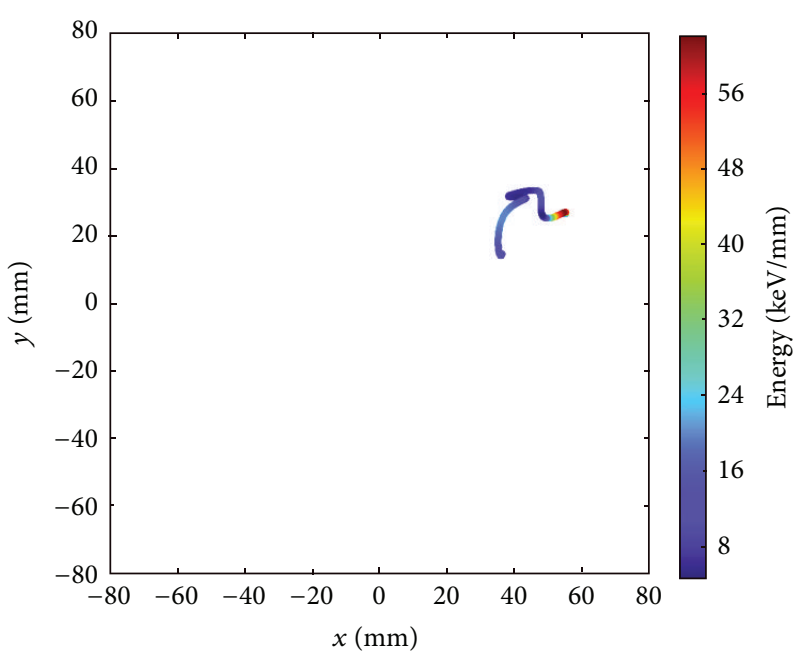

(b)

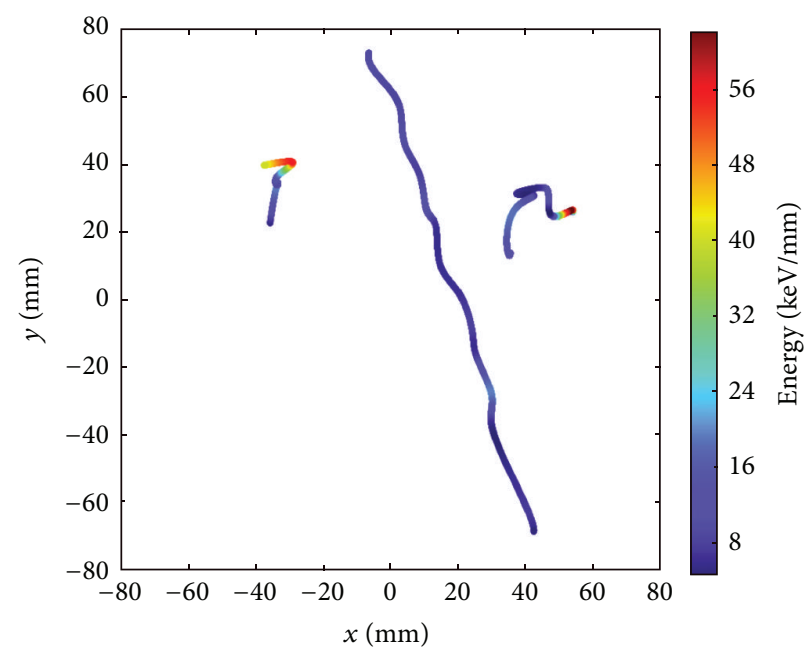

(d)

Figure 29: Examples of ${ }^{22} \mathrm{Na}$ (a), ${ }^{137} \mathrm{Cs}$ (b), and muon (c) track $x y$ plane projections. (d) The three events with the same energy scale. The endpoint of the electron is clearly visible for ${ }^{22} \mathrm{Na}$ and ${ }^{137} \mathrm{Cs}$ while the energy deposition for the muon is almost constant. Tracks reconstructed from NEXT-DEMO data [41].

with a clearly visible endpoint at the end of the track with a higher energy deposition (blob). On the other hand, the reconstruction of a muon track shows a straight line through the detector with a fairly uniform energy deposition.

NEXT-DEMO has been running successfully for two years, proving perfect high voltage operation and a great stability against sparks. The gas system, completed with a hot getter, has demonstrated to be leakproof (less than $0.1 \%$ leakage per day) and has allowed a continuous recirculation and purification of the gas, which resulted in a measured electron lifetime of up to tens of milliseconds. The light collection efficiency has been thoroughly understood, by studies of both primary and electroluminescent scintillation signals. The TPB coating on the PTFE reflectors in the drift region produced an increase in the EL light collection of a factor of three [29], thus improving light statistics. Data produced with an alpha source have allowed studies of primary scintillation signals along the whole drift length, leading to a better understanding of light reflectance and loss in our detector, through the support of Monte Carlo simulations [39].

To summarise, the NEXT-DEMO detector is operating continuously at IFIC since 2011. The current configuration, with a SiPM tracking plane, a PMT energy plane, and a light tube coated with TPB, demonstrates the design chosen for the NEXT-100 detector, exercises all the technical solutions, and shows excellent energy resolution and electron reconstruction. Further work is currently in progress analysing the many millions of events acquired with the chamber.

5.2. NEXT-DBDM. The basic building blocks of the NEXTDBDM xenon electroluminescent TPC are shown in Figures 30 and 31: a stainless-steel pressure vessel, a gas system that recirculates and purifies the xenon at $10-15 \mathrm{~atm}$, stainless steel wire meshes that establish high-voltage equipotential planes 


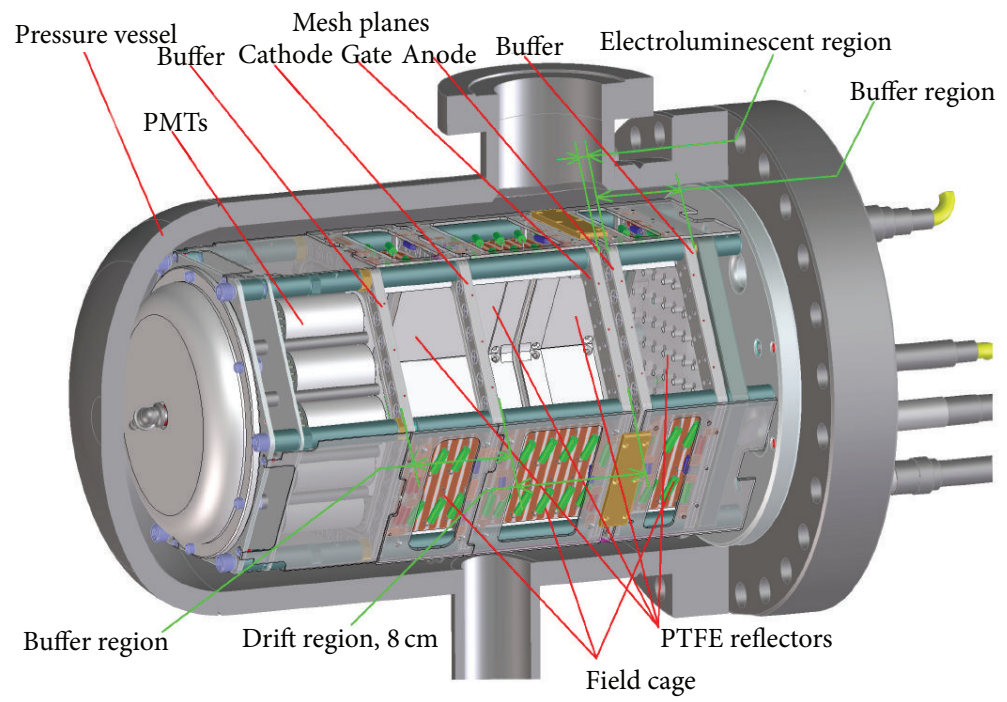

Figure 30: The NEXT-DBDM electroluminescent TPC configuration. An array of 19 photomultipliers (PMTs) measures S1 primary scintillation light from the $8 \mathrm{~cm}$ long drift region and S2 light produced in the $0.5 \mathrm{~cm}$ electroluminescence (EL) region. Two $5 \mathrm{~cm}$ long buffer regions behind the EL anode mesh and between the PMTs and the cathode mesh grade the high voltages (up to $\pm 17 \mathrm{kV}$ ) down to ground potential.

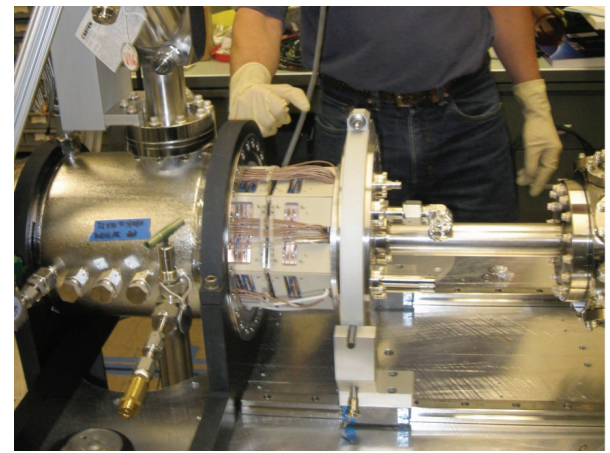

FIGURE 31: The NEXT-DBDM prototype, operating at LBNL. Insertion of the time projection chamber into the stainless-steel pressure vessel.

in the boundaries of the drift and the EL regions, field cages with hexagonal cross sections to establish uniform electric fields in those regions, a hexagonal pattern array of $19 \mathrm{VUV}$ sensitive PMTs inside the pressure vessel, and an associated readout electronics and data-acquisition system.

In the NEXT-DBDM detector the PMT array and the EL region, which are both hexagonal areas with $12.4 \mathrm{~cm}$ between opposite sides, are $13.5 \mathrm{~cm}$ away from each other. Thus, pointlike isotropic light produced in the EL region illuminates the PMT array with little PMT-to-PMT variation. This geometric configuration also makes the illumination pattern and the total light collection only very mildly dependent on the position of the light origin within the EL region. The diffuse reflectivity of the TPC walls increases this light collection uniformity further. As a result, the device provides good energy measurements with little dependence on the position of the charge depositions. On the other hand, without a light sensor array near the EL region precise tracking information is not available. Still, the position reconstruction achievable allows the fiducialization of pulses to select events/pulses within regions of the TPC with uniform light collection efficiencies.

The field configuration in the TPC is established by five stainless steel meshes with $88 \%$ open area at a $z$ position of $0.5 \mathrm{~cm}$ (cathode buffer or PMT mesh), $5.5 \mathrm{~cm}$ (cathode or drift start mesh), $13.5 \mathrm{~cm}$ (field transition or EL-start mesh), $14.0 \mathrm{~cm}$ (anode or EL-end mesh), and $19.0 \mathrm{~cm}$ (anode buffer or ground mesh) from the PMT windows. Electroluminescence occurs between 13.5 and $14.0 \mathrm{~cm}$. The meshes are supported and kept tense by stainless steel frames made out of two parts and tensioning screws on the perimeter. The TPC side walls, made out of 18 individual rectangular assemblies $7.1 \mathrm{~cm}$ wide (and 5 and $8 \mathrm{~cm}$ long) connecting adjacent meshes (except around the $0.5 \mathrm{~cm}$ EL gap), serve the dual purpose of light cage and field cage. Each side wall assembly is made of a $0.6 \mathrm{~cm}$ thick PTFE panel and a ceramic support panel. The PTFE panels are bare on the side facing the active volume and have copper stripes parallel to the mesh planes every $0.6 \mathrm{~cm}$ on the other side. The bare PTFE serves as reflector for the VUV light. Adjacent copper stripes are linked with $100 \mathrm{M} \Omega$ resistors to grade the potential and produce a uniform electric field. The ceramic support panels are connected, mechanically and electrically, to the outer perimeter of the mesh support frames and to the first and last copper stripes on their corresponding PTFE panel. High voltage connections to establish the TPC fields (HHV) are made directly to the mesh frames.

In Figure 32 the energy spectrum in the $662 \mathrm{keV}$ full energy region obtained at $10 \mathrm{~atm}$ is shown. A 1.1\% FWHM energy resolution was obtained for events reconstructed in the central $0.6 \mathrm{~cm}$ radius region. A small drift-time dependent correction for attachment losses with $\tau=13.9 \mathrm{~ms}$ was applied. The xenon X-ray escape peak is clearly visible, 


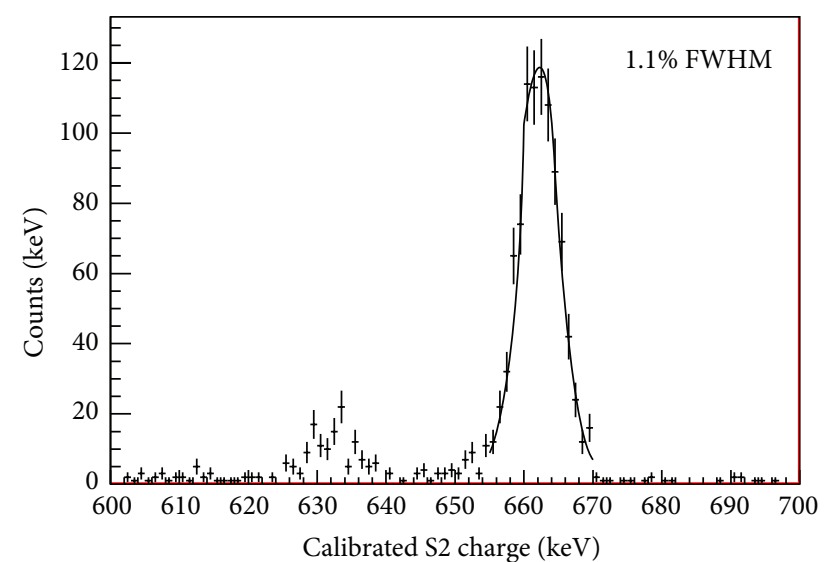

FIGURE 32: Energy resolution at $10 \mathrm{~atm}$ for $662 \mathrm{keV}$ gamma rays [38].

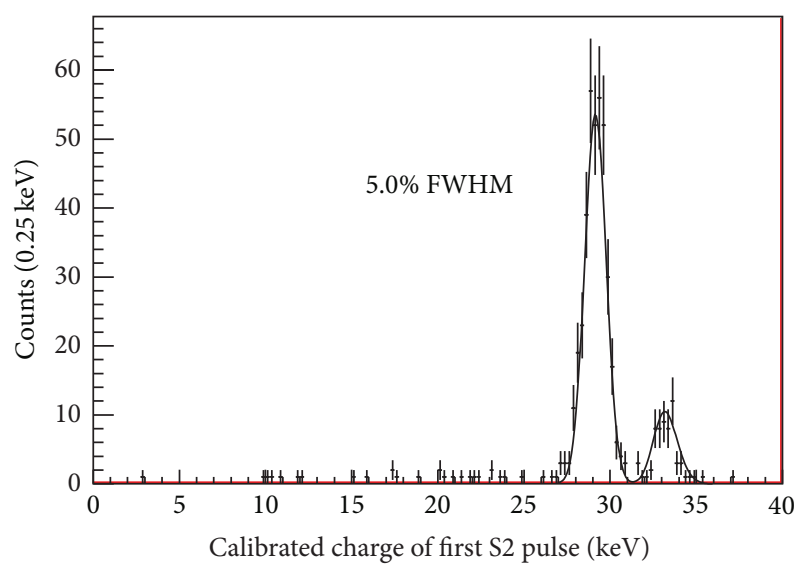

FIgURE 33: Energy resolution at 10 atm for $30 \mathrm{keV}$ xenon X-rays [38].

$\sim 30 \mathrm{keV}$ below the main peak. For the spectrum taken at $15 \mathrm{~atm}$ a $1 \%$ FWHM resolution was obtained. This resolution extrapolates to $0.52 \%$ FWHM at $Q_{\beta \beta}=2.458 \mathrm{MeV}$ if the scaling follows a statistical $1 / \sqrt{E}$ dependence and no other systematic effect dominates.

In order to study the EL TPC energy resolution at lower energies, full energy $662 \mathrm{keV}$ events that had a well separated $\mathrm{X}$-ray pulse reconstructed in the central $1.5 \mathrm{~cm}$ radius region were used. Events with only an X-ray deposition are difficult to trigger, because of the low energy. However, X-ray depositions are also present in events with the photoelectron fully contained and the $30 \mathrm{keV}$ disexcitation X-ray well separated. Figure 33 shows the energy spectrum obtained at 10 atm with a 5\% FWHM resolution.

Figure 34 summarises our measurements and understanding of the EL TPC energy resolution. The lower diagonal line represents the Poisson statistical limit from the measurement of a small fraction of the photons produced by the EL gain while the upper diagonal line includes the degradation (mostly from PMT afterpulsing) due to PMT response. The circle data points show the energy resolutions obtained for dedicated LED runs with varying light intensities per LED pulse. The LED points follow the expected resolution

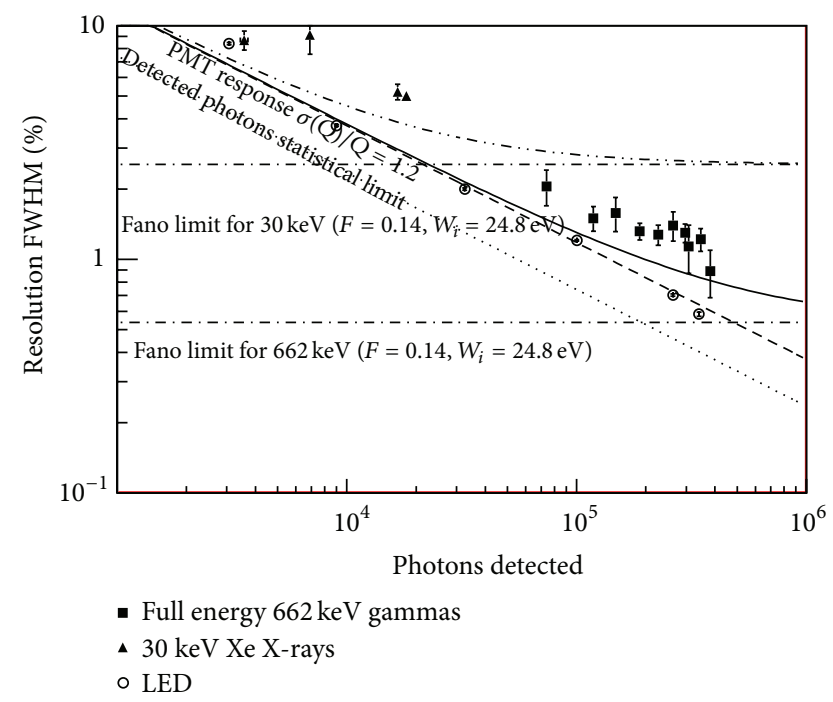

FIGURE 34: Energy resolution in the high-pressure xenon NEXT-DBDM electroluminescent TPC. Data points show the measured energy resolution for $662 \mathrm{keV}$ gammas (squares), $\sim 30 \mathrm{keV}$ xenon X-rays (triangles), and LED light pulses (circles) as a function of the number of photons detected. The expected resolution including the intrinsic Fano factor, the statistical fluctuations in the number of detected photons, and the PMT charge measurement variance is shown for X-rays (dot dot dashed) and for $662 \mathrm{keV}$ gammas (solid line). Resolutions for the $662 \mathrm{keV}$ peak were obtained from $15.1 \mathrm{~atm}$ data runs while $\mathrm{X}$-ray resolutions were obtained from $10.1 \mathrm{~atm}$ runs [38]

over the two-decade range studied. The two horizontal lines represent the xenon gas nominal intrinsic resolution for 30 and $662 \mathrm{keV}$, respectively, and the two curved lines are the expected EL TPC resolutions with contributions from the intrinsic limit and the photons' measurement. Our $662 \mathrm{keV}$ data (squares) and xenon X-ray data (triangles) taken with various EL gains follow the expected functional form of the resolution but are $20-30 \%$ larger possibly due to the $x y$ response nonuniformity. Detailed track imaging from a dense photosensor array near the EL region, such as the one recently commissioned for the NEXT-DBDM prototype, will enable the application of $x y$ position corrections to further improve the energy measurement.

\section{Conclusions}

In this paper, the current status and future prospects of the NEXT project and in particular of the NEXT-100 experiment at LSC have been described. NEXT has a large discovery potential and the capability to offer a technique that can be extrapolated, at a very competitive cost, to the ton scale.

The collaboration has developed the advanced technology of high-pressure chambers, and, in particular, NEXT-DEMO is the first large-scale HPXe TPC using the EL technology. The innovations include the use of SiPMs for the tracking plane, a technology that was in its infancy only five years ago.

The collaboration has published results that illustrate the physics case and demonstrate the good performance of the EL 
technology. Very good energy resolution, better than $1 \%$ FWHM, has been measured and the topological signature of electrons has been clearly established.

The NEXT-100 detector is now in the initial stages of construction and is expected to be taking data in 2015 . The scientific opportunity is extraordinary and the cost to scientific impact ratio is relatively modest. In addition to contributing to the current scientific development, NEXT could be the springboard for a future, large-scale experiment that could finally demonstrate that the Ettore Majorana insight was correct.

\section{Conflict of Interests}

The authors declare that there is no conflict of interests regarding the publication of this paper.

\section{Acknowledgments}

This work was supported by the following agencies and institutions: the Ministerio de Economía y Competitividad of Spain under Grants CONSOLIDER-Ingenio 2010 CSD20080037 (CUP), FPA2009-13697-C04-04, and FIS2012-37947C04; the Director, Office of Science, Office of Basic Energy Sciences of the US Department of Energy under Contract no. DE-AC02-05CH11231; and the Portuguese FCT and FEDER through the program COMPETE, Projects PTDC/FIS/103860/2008 and PTDC/FIS/112272/2009. J. Renner (LBNL) acknowledges the support of a US DOE NNSA Stewardship Science Graduate Fellowship under Contract no. DE-FC52-08NA28752. J. J. Gómez-Cadenas is a spokes person.

\section{References}

[1] M. Auger, D. J. Auty, P. S. Barbeau et al., "Search for neutrinoless double-beta decay in ${ }^{136}$ Xe with EXO-200," Physical Review Letters, vol. 109, no. 3, Article ID 032505, 6 pages, 2012.

[2] A. Gando, Y. Gando, H. Hanakago et al., "Limit on neutrinoless $\beta \beta$ decay of ${ }^{136} \mathrm{Xe}$ from the first phase of KamLAND-Zen and comparison with the positive claim in ${ }^{76} \mathrm{Ge}$," Physical Review Letters, vol. 110, no. 6, Article ID 062502, 5 pages, 2013.

[3] P. A. R. Ade, N. Aghanim, C. Armitage-Caplan et al., "Planck 2013 results. XVI. cosmological parameters," http://arxiv.org/abs/1303.5076.

[4] N. Haba and R. Takahashi, "Constraints on neutrino mass ordering and degeneracy from Planck and neutrino-less double beta decay," http://arxiv.org/abs/1305.0147.

[5] E. Majorana, "Theory of the symmetry of electrons and positrons," Il Nuovo Cimento, vol. 14, no. 4, pp. 171-184, 1937.

[6] P. Hernández, "Neutrino physics," CERN Yellow Report CERN 2010-001, 2010.

[7] M. Fukugita and T. Yanagida, "Barygenesis without grand unification," Physics Letters B, vol. 174, no. 1, pp. 45-47, 1986.

[8] S. Davidson, E. Nardi, and Y. Nir, "Leptogenesis," Physics Reports, vol. 466, no. 4-5, pp. 105-177, 2008.

[9] H. V. Klapdor-Kleingrothaus, A. Dietz, L. Baudis et al., "Latest results from the Heidelberg-Moscow double beta decay experiment," European Physical Journal A, vol. 12, no. 2, pp. 147-154, 2001.
[10] H. V. Klapdor-Kleingrothaus and I. V. Krivosheina, "The evidence for the observation of $0 \nu \beta \beta$ decay: the identification of $0 \nu \beta \beta$ events from the full spectra," Modern Physics Letters A, vol. 21, no. 20, pp. 1547-1566, 2006.

[11] C. M. Cattadori, "GERDA status report: results from commissioning," Journal of Physics, vol. 375, no. 4, Article ID 042008, 2012.

[12] J. Wilkerson, E. Aguayo, F. Avignone, H. Back, A. Barabash et al., "The majorana demonstrator: a search for neutrinoless doublebeta decay of germanium-76," Journal of Physics, vol. 375, no. 4, Article ID 042010, 2012.

[13] P. Gorla, "The CUORE experiment: status and prospects," Journal of Physics, vol. 375, no. 4, Article ID 042013, 2012.

[14] J. J. Gómez-Cadenas, J. Martín-Albo, M. Sorel et al., "Sense and sensitivity of double beta decay experiments," Journal of Cosmology and Astroparticle Physics, vol. 2011, no. 6, article 007, 2011.

[15] X. Sarazin, "Review of double beta experiments," http://arxiv.org/abs/1210.7666.

[16] J. Maneira, "The SNO+ experiment: status and overview," Journal of Physics, vol. 447, no. 1, Article ID 012065, 2013.

[17] J. J. Gómez-Cadenas, J. Martín-Albo, M. Mezzetto, F. Monrabal, and M. Sorel, "The search for neutrinoless double beta decay," Rivista del Nuovo Cimento, vol. 35, no. 2, pp. 29-98, 2012.

[18] O. Cremonesi, "Experimental searches of neutrinoless double beta decay," Nuclear Physics B, vol. 237-238, pp. 7-12, 2013.

[19] A. Giuliani and A. Poves, "Neutrinoless double-beta decay," Advances in High Energy Physics, vol. 2012, Article ID 857016, 38 pages, 2012.

[20] K. Zuber, "Neutrinoless double beta decay experiments," Acta Physica Polonica B, vol. 37, no. 7, Article ID 061000, pp. 19051921, 2006.

[21] M. Auger, D. Auty, P. Barbeau, L. Bartoszek, E. Baussan et al., "The EXO-200 detector, part I: detector design and construction," Journal of Instrumentation, vol. 7, no. 5, Article ID P05010, 2012.

[22] A. Gando, Y. Gando, H. Hanakago et al., "Measurement of the double- $\beta$ decay half-life of ${ }^{136}$ Xe with the KamLAND-Zen experiment," Physical Review C, vol. 85, no. 4, Article ID 045504, 2012.

[23] V. Álvareza, F. I. G. M. Borgesb, S. Cárcela et al., "NEXT-100 technical design report (TDR): executive summary," Journal of Instrumentation, vol. 7, no. 6, Article ID T06001, 2012.

[24] J. Albert, M. Auger, D.J. Auty et al., "An improved measurement of the $2 \nu \beta \beta$ half-life of ${ }^{136} \mathrm{Xe}$ with EXO-200," http://arxiv.org/abs/1306.6106.

[25] Caltech-Neuchâtel-PSI Collaboration, R. Luscher et al., "Search for $\beta \beta$ decay in ${ }^{136} \mathrm{Xe}$ : new results from the Gotthard experiment," Physics Letters B, vol. 434, no. 3-4, pp. 407-414, 1998.

[26] E. Conti, R. Devoe, G. Gratta et al., "Correlated uctuations between luminescence and ionization in liquid xenon," Physical Review B, vol. 68, Article ID 054201, 5 pages, 2003.

[27] J. Bergstrom, "Combining and comparing neutrinoless double beta decay experiments using different nuclei," Journal of High Energy Physics, vol. 2013, no. 2, article 93, 2013.

[28] J. Gómez-Cadenas, J. Martín-Albo, J. Munoz Vidal, and C. PenaGaray, "Discovery potential of xenon-based neutrinoless double beta decay experiments in light of small angular scale CMB observations," Journal of Cosmology and Astroparticle Physics, vol. 2013, no. 3, article 043, 2013. 
[29] V. Álvarez, F. I. G. M. Borges, S. Cárcel et al., "Initial results of NEXT-DEMO, a large-scale prototype of the NEXT-100 experiment," Journal of Instrumentation, vol. 8, no. 5, Article ID P04002, 2013.

[30] J. Martín-Albo and J. J. Gómez-Cadenas, "Status and physics potential of NEXT-100," Journal of Physics, vol. 460, no. 1, Article ID 012010, 2013.

[31] D. Nygren, "High-pressure xenon gas electroluminescent TPC for 0- $\nu \beta$-decay search," Nuclear Instruments and Methods in Physics Research Section A, vol. 603, no. 3, pp. 337-348, 2009.

[32] V. Álvarez, M. Ball, M. Batallé et al., “The NEXT-100 experiment for neutrinoless double beta decay searches (conceptual design report)," http://arxiv.org/abs/1106.3630.

[33] K. Lung, K. Arisaka, A. Bargetzi et al., "Characterization of the Hamamatsu R11410-10 3-in. photomultiplier tube for liquid xenon dark matter direct detection experiments," Nuclear Instruments and Methods in Physics Research Section A, vol. 696, pp. 32-39, 2012.

[34] R. Collaboration, "Development of micro-pattern gas detectors technologies," http://rd51-public.web.cern.ch/rd51-public/.

[35] V. Alvarez, I. Bandac, A. Bettini et al., "Radiopurity control in the NEXT-100 double beta decay experiment: procedures and initial measurements," Journal of Instrumentation, vol. 8, Article ID T01002, 2012.

[36] E. Aprile, K. Arisaka, F. Arneodo et al., "Material screening and selection for XENON100," Astroparticle Physics, vol. 35, no. 2, pp. 43-49, 2011.

[37] S. Agostinelli, J. Allisonas, K. Amakoe et al., "GEANT4- a simulation toolkit," Nuclear Instruments and Methods in Physics Research Section A, vol. 506, no. 3, pp. 250-303, 2003.

[38] V. Álvarez, F. I. G. M. Borgesb, S. Cárcela et al., "Nearintrinsic energy resolution for 30-662 keV gamma rays in a high pressure xenon electroluminescent TPC," Nuclear Instruments and Methods in Physics Research Section A, vol. 708, pp. 101-114, 2012.

[39] V. Álvarez, F. I. G. M. Borges, S. Cárcel et al., "Ionization and scintillation response of high-pressurexenon gas to alpha particles," Journal of Instrumentation, vol. 8, no. 5, Article ID P05025, 2013.

[40] C. Silva, J. Pinto da Cunha, A. Pereira, V. Chepel, M. Lopes et al., "Reflectance of polytetrafluoroethylene (PTFE) for xenon scintillation light," Journal of Applied Physics, vol. 107, no. 6, pp. 064902-064908, 2010.

[41] V. Álvarez, F.I.G. Borges, S. Cárcel et al., "Operation and first results of the NEXT-DEMO prototype using a silicon photomultiplier tracking array," Journal of Instrumentation, vol. 8, no. 9, Article ID P09011, 2013. 

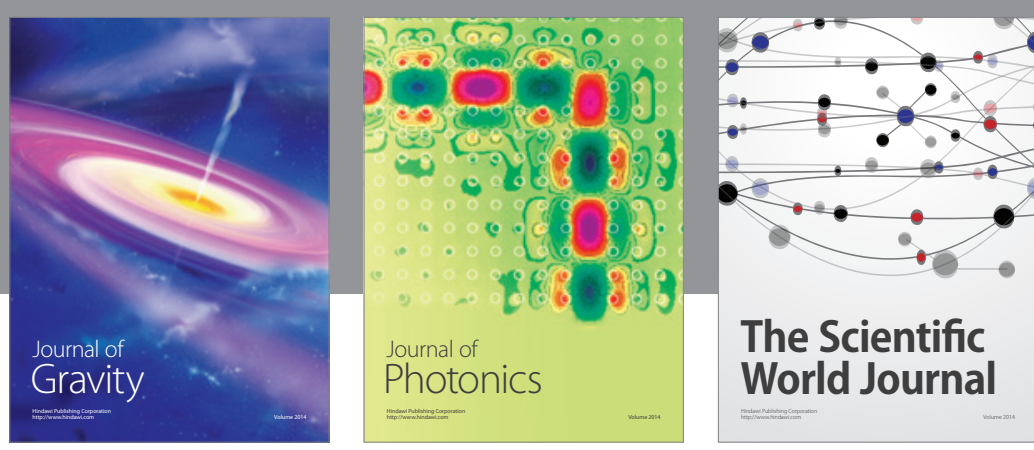

The Scientific World Journal
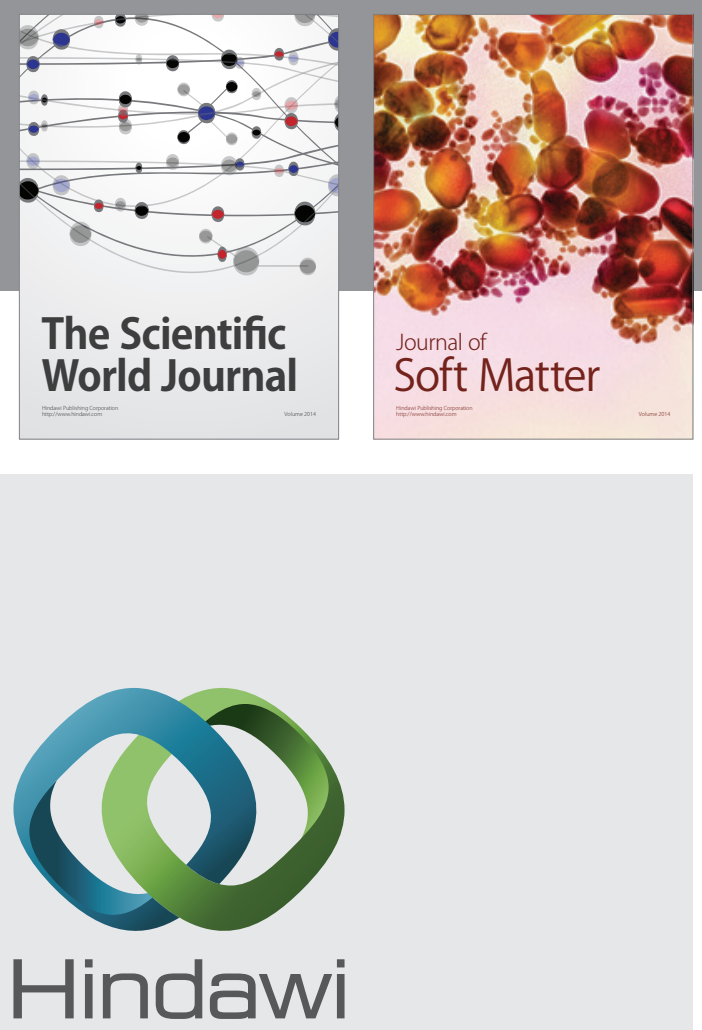

Submit your manuscripts at

http://www.hindawi.com

nternational Journal of

Statistical Mechanics
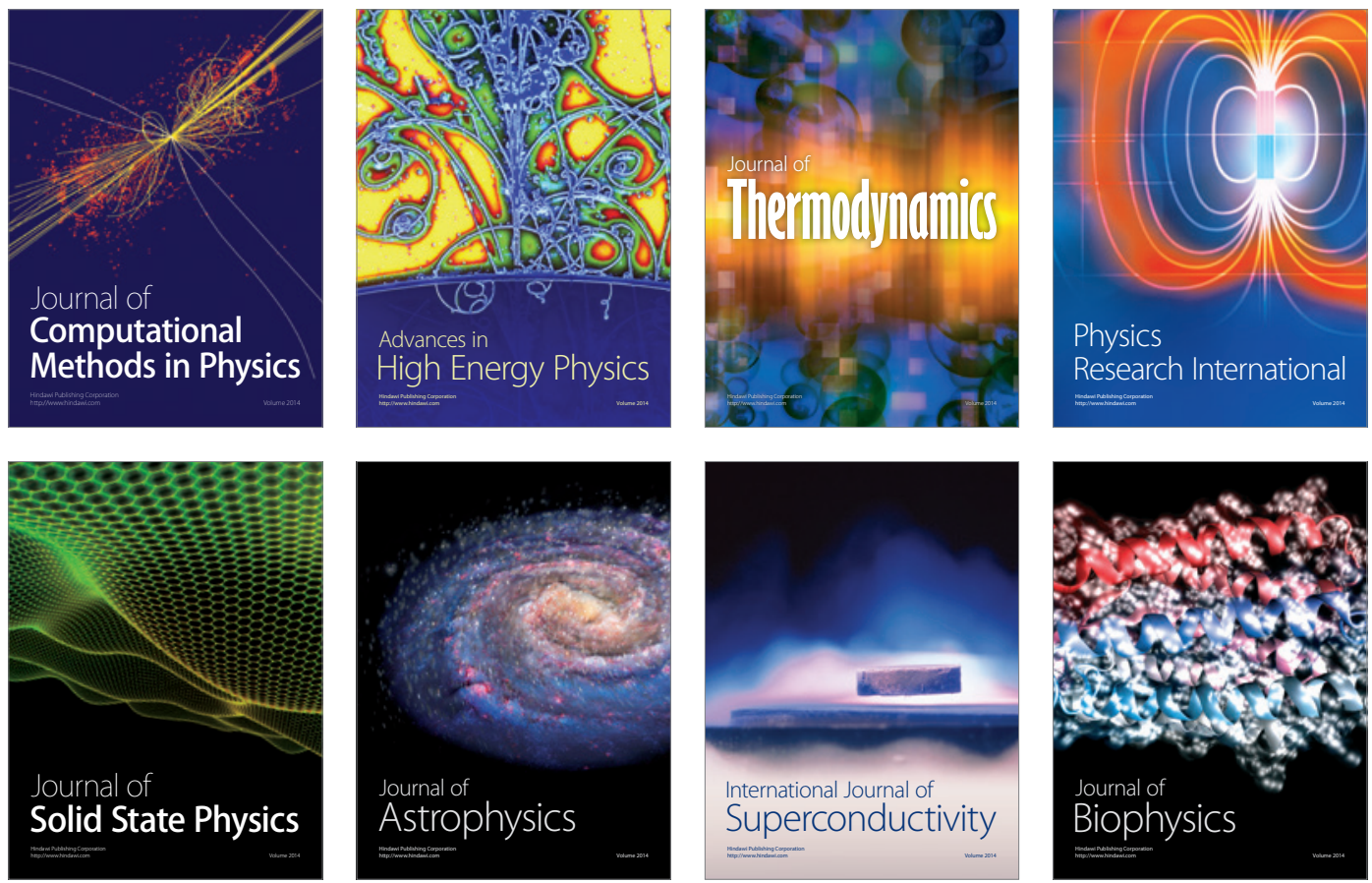
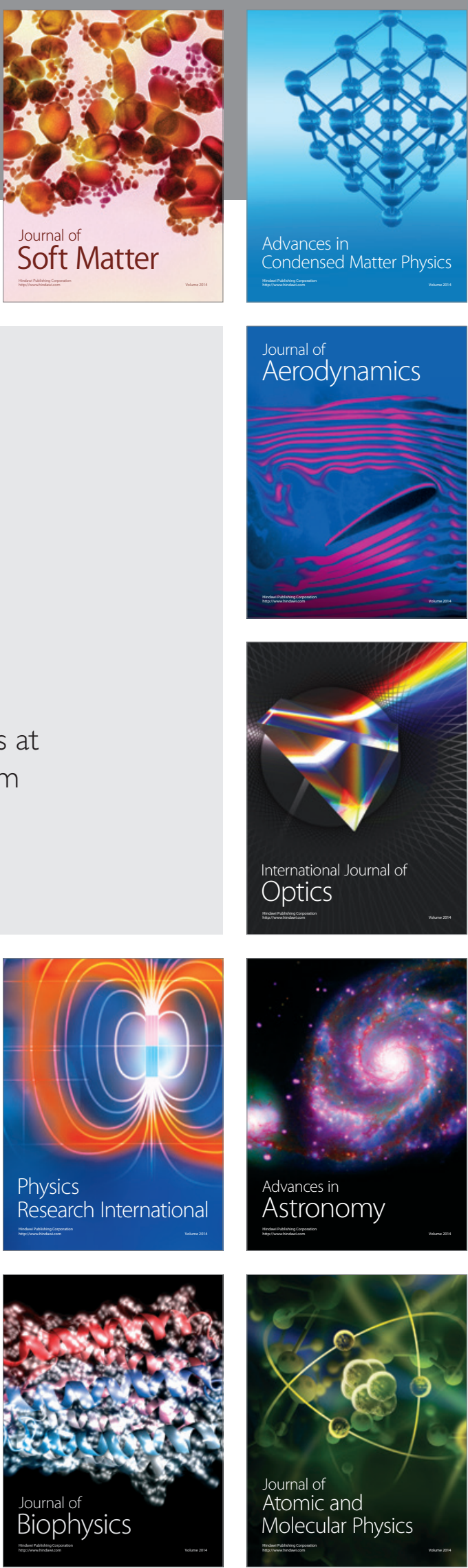